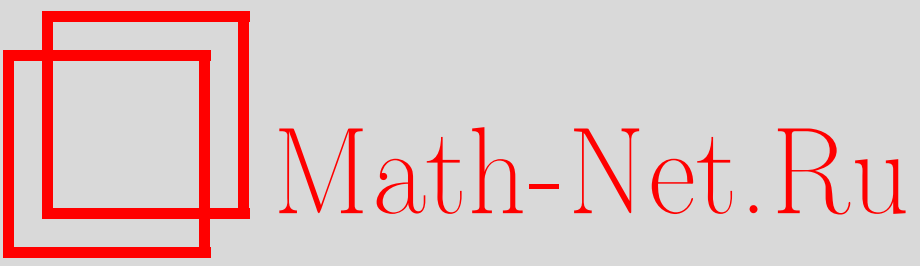

В. А. Ватутин, Системы поллинга и многотипные ветвящиеся процессы в случайной среде с финальным продуктом, Теория вероятн. и ее примен., 2010, том 55, выпуск 4, 644-679

DOI: https://doi.org/10.4213/tvp4277

Использование Общероссийского математического портала Math-Net.Ru подразумевает, что вы прочитали и согласны с пользовательским соглашением

http: //www . mathnet.ru/rus/agreement

Параметры загрузки:

IP : 54.210 .77 .194

26 апреля 2023 г., 17:05:56 
ТЕОРИЯ ВЕРОЯТНОСТЕЙ

Том 55

И ЕЕ ПРИМЕНЕНИЯ

Выпуск 4

2010

(C) $2010 \Gamma$.

ВАТУТИН В. А.*

\title{
СИСТЕМЫ ПОЛЛИНГА И МНОГОТИПНЫЕ ВЕТВЯЩИЕСЯ ПРОЦЕССЫ В СЛУЧАЙНОЙ СРЕДЕ С ФИНАЛЬНЫМ ПРОДУКТОМ ${ }^{1)}$
}

\begin{abstract}
Методами многотипных ветвящихся процессов в случайной среде с финальными продуктом мы исследуем асимптотику хвостов распределений длин периодов занятости и некоторых других характеристик систем поллинга ветвящегося типа, в которых дисциплины обслуживания, входные параметры и распределения времен обслуживания меняются случайным образом.
\end{abstract}

Ключевые слова и фразы: системы поллинга, многотипные ветвящиеся процессы в случайной среде, финальный продукт, период занятости, случайные матрицы.

1. Системы поллинга с дисциплинами обслуживания ветвящегося типа. Рассмотрим систему поллинга, состоящую из одного обслуживающего прибора и $m$ станций, занумерованных числами $i \in\{1, \ldots, m\}$, каждая из которых имеет бесконечное число мест ожидания. Первоначально в системе нет требований. Как только в систему поступают требования, прибор немедленно начинает обслуживание, посещая станции в циклическом порядке $(1 \rightarrow 2 \rightarrow \cdots \rightarrow m \rightarrow 1 \rightarrow \cdots)$, начиная со станции 1 , в соответствии с выбранной дисциплиной обслуживания (которая будет описана позднее) и с мгновенным переключением от очереди к очереди. В дальнейшем первоначальный отрезок обслуживания по маршруту $(1 \rightarrow 2 \rightarrow \cdots \rightarrow m)$ мы будем называть нулевым циклом. Последующие отрезки обслуживания такого типа будут называться первым циклом, вторым циклом и т.д. Если в системе нет требований, то прибор ожидает их прихода на парковке, обозначаемой символом R. Требования прибывают в систему в соответствии с точечным процессом, параметры которого меняются случайным образом при переключении прибора со станции на станцию.

\footnotetext{
*Математический институт им. В.А.Стеклова РАН, ул. Губкина, 8, 119991 Москва, Россия; e-mail: vatutin@mi.ras.ru

1) Работа выполнена при поддержке РФФИ (грант 08-01-00078) и программы РАН «Математическая теория управления».
} 
Для строгого описания процесса поступления требований и порядка их обслуживания в интересующей нас системе нам понадобится ряд понятий.

Пусть $\mathbf{s}:=\left(s_{1}, \ldots, s_{m}\right) \in[0,1]^{m}$ обозначает $m$-мерную переменную,

$$
\mathbf{s}^{\mathbf{k}}:=s_{1}^{k_{1}} \cdots s_{m}^{k_{m}}, \quad k_{i} \in \mathbb{N}_{0}=\{0,1,2, \ldots\},
$$

и пусть $\mathbf{e}_{i}:=(0, \ldots, 1, \ldots, 0)-m$-мерный вектор, $i$-я координата которого равна 1 , а все остальные равны 0 . Наконец, символы $\mathbf{0}$ и $\mathbf{1}$ будут соответствовать $m$-мерным векторам, все координаты которых равны 0 и 1 соответственно. Обозначим

$$
\chi^{(i)}(\mathbf{s} ; \lambda):=\mathbf{E}\left[s_{1}^{\theta_{i 1}} \cdots s_{m}^{\theta_{i m}} e^{-\lambda \phi_{i}}\right], \quad i=1, \ldots, m,
$$

смешанные вероятностные производящие функции (с.в.п.ф.) $(m+1)$ мерных векторов $\left(\theta_{i 1}, \ldots, \theta_{i m} ; \phi_{i}\right)$, где $\theta_{i j}$ - неотрицательные целочисленные случайные величины, а $\phi_{i}$ - неотрицательная случайная величина. Пусть, далее, $\chi(\mathbf{s} ; \lambda)=\left(\chi^{(1)}(\mathbf{s} ; \lambda), \ldots, \chi^{(m)}(\mathbf{s} ; \lambda)\right)-$ соответствующие векторнозначные с.в.п.ф., и пусть $\mathscr{X}=\{\chi(\mathbf{s} ; \lambda)\}$ - множество всех таких с.в.п.ф. Предположим, что на элементах естественной $\sigma$-алгебры выборочного пространства $\mathscr{X}$ задана вероятностная мера $\mathbb{P}$, а

$$
\chi_{0}(\mathbf{s} ; \lambda), \chi_{1}(\mathbf{s} ; \lambda), \chi_{2}(\mathbf{s} ; \lambda), \ldots,
$$

- последовательность с.в.п.ф., выбранных из $\mathscr{X}$ независимым образом в соответствии с мерой $\mathbb{P}$, где векторнозначная функция $\chi_{n}(\mathbf{s} ; \lambda):=$ $\left(\chi_{n}^{(1)}(\mathbf{s} ; \lambda), \ldots, \chi_{n}^{(m)}(\mathbf{s} ; \lambda)\right)$ имеет компоненты

$$
\chi_{n}^{(i)}(\mathbf{s} ; \lambda):=\mathbf{E}\left[s_{1}^{\theta_{i 1}(n)} \cdots s_{m}^{\theta_{i m}(n)} e^{-\lambda \phi_{i}(n)}\right] .
$$

В данной работе мы изучаем системы поллинга, потоки требований и дисциплины обслуживания которых удовлетворяют следующему условию.

Свойство ветвления. Если прибор, прибывший для обслуживания на станцию $i$ в $n$-й раз, обнаружит там, скажем, $k_{i}$ требований, занумерованных для определенности числами $1, \ldots, k_{i}$, то во время этого визита процесс поступления новых требований устроен таким образом, что в конце каждого этапа обслуживания требования $j$ (количество таких этапов может быть отлично от единицы, если дисциплина обслуживания допускает повторное обслуживание в течение одного визита) очереди в системе будут увеличены на случайный набор требований $\left(\theta_{i 1}(n, j), \ldots, \theta_{i m}(n, j)\right)$, где $\theta_{i l}(n, j)$ - количество требований, пришедших на станцию $l$, и, кроме того, финальный продукт объема $\phi_{i}(n, j) \geqslant 0$ будет добавлен в систему. При этом предполагается, что 
$\left(\theta_{i 1}(n, j), \ldots, \theta_{i m}(n, j) ; \phi_{i}(n, j)\right), j=1, \ldots, k_{i},-$ независимые одинаково распределенные векторы, причем

$$
\mathbf{E}\left[s_{1}^{\theta_{i 1}(n, j)} \cdots s_{m}^{\theta_{i m}(n, j)} e^{-\lambda \phi_{i}(n, j)}\right]=\chi_{n}^{(i)}(\mathbf{s} ; \lambda) .
$$

Заметим, что с.в.п.ф. $\chi_{n}^{(i)}(\mathbf{s} ; \lambda)$ случайна и, следовательно, параметры данной системы поллинга меняются от цикла к циклу случайным образом. Поскольку в данной работе нас будут интересовать характеристики, связанные с периодами занятости системы, работа которой начинается с поступления в систему одного требования в момент 0 на станцию $J \in\{1, \ldots, m\}$, то порядок поступления требований в систему, свободную от требований, не имеет значения для последующих рассуждений.

Целью данной работы является изучение распределения совокупного размера финального продукта, накопленного в системе за время периода занятости прибора. В частности, для случая, когда в качестве финального продукта $\phi_{i}(n, j)$ выступает время обслуживания $j$-го требования, поступившего на обслуживание во время $n$-го визита прибора на станцию $i$, будут указаны условия на характеристики системы, при выполнении которых хвост распределения длины периода занятости убывает при $y \rightarrow \infty$ как const $\cdot y^{-\kappa}$ для некоторого $\kappa>0$.

Прежде чем переходить к точным формулировкам наших результатов, рассмотрим два примера систем поллинга, обладающих свойством ветвления.

Обозначим $\mathscr{T}_{+}=\{T\}$ множество всех распределений неотрицательных случайных величин, и пусть $\mathscr{T}_{+}^{m}:=\left\{\left(T_{1}, \ldots, T_{m}\right): T_{i} \in \mathscr{T}_{+}\right\}-$множество $m$-мерных наборов таких распределений, $\mathscr{M}_{\varepsilon}=\{\mathscr{E}\}-$ множество матриц $\mathscr{E}=\left(\varepsilon_{i j}\right)_{i, j=1}^{m}$ размера $m \times m$ с неотрицательными элементами, а $\mathscr{M}_{\gamma}=\{\Gamma\}-$ множество всех матриц $\Gamma=\left(\gamma_{i j}\right)_{i=1, j=0}^{m}$ размера $m \times(m+1)$ с неотрицательными элементами такими, что

$$
\sum_{j=0}^{m} \gamma_{i j}=1, \quad i=1, \ldots, m, \quad \text { и } \quad \max _{1 \leqslant i \leqslant m} \gamma_{i 0}>0 .
$$

Пусть $P_{\varepsilon}$ - мера на борелевской $\sigma$-алгебре пространства $\mathscr{M}_{\varepsilon} \times \mathscr{M}_{\gamma} \times \mathscr{T}_{+}^{m}$.

П р и м е р 1 (по мотивам работы [34]). Рассмотрим систему поллинга с $m$ станциями и одним прибором, совершающим циклическое обслуживание требований на станциях. Допустим, что в начальный момент требований в системе нет, а прибор расположен на парковке $R$. Предположим, что при отсутствии требований в системе, требования поступают на станцию $i$ в соответствии с пуассоновским потоком неслучайной интенсивности $\varepsilon_{i}$. В момент появления первого требования в системе прибор выбирает случайный элемент $\left(\mathscr{E}_{0}, \Gamma_{0}, \mathbf{T}_{0}\right) \in \mathscr{M}_{\varepsilon} \times \mathscr{M}_{\gamma} \times \mathscr{T}_{+}^{m}$, 
где

$$
\mathscr{E}_{0}=\left(\varepsilon_{i j}(0)\right)_{i, j=1}^{m}, \quad \Gamma_{0}=\left(\gamma_{i j}(0)\right)_{i=1, j=0}^{m}, \quad \mathbf{T}_{0}=\left(T_{10}, \ldots, T_{m 0}\right)
$$

и немедленно начинает нулевой цикл обслуживания по маршруту $(1 \rightarrow$ $2 \rightarrow \cdots \rightarrow m)$ в соответствии со шлюзовой дисциплиной с обратной связью. А именно, прибор обслуживает все требования, находившиеся на станции в момент его прибытия, а затем мгновенно переходит на обслуживание требований на следующей (по циклу) станции. В промежутке времени, когда прибор обслуживает группу требований на станции $i$, новые требования поступают в систему в соответствии с независимыми пуассоновскими потоками с интенсивностями, задаваемыми вектором $\left(\varepsilon_{i 1}(0), \ldots, \varepsilon_{i m}(0)\right)$ (некоторые компоненты могут быть равны нулю), а времена обслуживания требований независимы и имеют одну и ту же функцию распределения $T_{i 0}(x):=\mathbf{P}\left(\tau_{i}(0) \leqslant x\right)$. Обслуженное требование либо переходит на станцию $j \in\{1, \ldots, m\}$ с вероятностью $\gamma_{i j}(0)$, либо покидает систему с вероятностью $\gamma_{i 0}(0)$, независимо от остальных событий. Кроме того, после окончания каждого периода обслуживания какого-либо требования это требование добавляет в систему время своего обслуживания в качестве финального продукта. Предполагается, что при фиксированном наборе $\left(\mathscr{E}_{0}, \Gamma_{0}, \mathbf{T}_{0}\right)$ времена обслуживания и процесс поступления новых требований независимы.

Последующие циклы обслуживания с номерами $n=1,2, \ldots$ имеют такую же вероятностную структуру, задаваемую наборами

$$
\mathscr{E}_{n}=\left(\varepsilon_{i j}(n)\right)_{i, j=1}^{m}, \quad \Gamma_{n}=\left(\gamma_{i j}(n)\right)_{i=1, j=0}^{m}, \quad \mathbf{T}_{n}=\left(T_{1 n}, \ldots, T_{m n}\right)
$$

с той лишь разницей, что в начале цикла $n$ в системе может находиться более одного требования.

Покажем, что эта система обладает свойством ветвления. С этой целью обозначим

$$
t_{i n}(\lambda):=\int_{0}^{\infty} e^{-\lambda x} d T_{i n}(x)
$$

преобразование Лапласа распределения $T_{i n}(x)$ случайной величины $\tau_{i}(n)$, представляющей время обслуживания требования на станции $i$ во время $n$-го визита прибора.

Нетрудно проверить, что с.в.п.ф. $\boldsymbol{\chi}_{n}(\mathbf{s} ; \lambda)$ имеет следующие компоненты (в наших условиях и с временем обслуживания требования в качестве финального продукта):

$$
\begin{aligned}
\chi_{n}^{(i)}(\mathbf{s} ; \lambda) & :=\mathbf{E}\left[s_{1}^{\theta_{i 1}(n)} \cdots s_{m}^{\theta_{i m}(n)} e^{-\lambda \tau_{i}(n)}\right] \\
& =\int_{0}^{\infty} \mathbf{E}\left[s_{1}^{\theta_{i 1}(n)} \cdots s_{m}^{\theta_{i m}(n)} \mid \tau_{i}(n)=x\right] e^{-\lambda x} d T_{i n}(x)
\end{aligned}
$$




$$
\begin{aligned}
& =\left(\gamma_{i 0}(n)+\sum_{j=1}^{m} \gamma_{i j}(n) s_{j}\right) \int_{0}^{\infty} \prod_{j=1}^{m} e^{\varepsilon_{i j}(n)\left(s_{j}-1\right) x} e^{-\lambda x} d T_{i n}(x) \\
& =\left(\gamma_{i 0}(n)+\sum_{j=1}^{m} \gamma_{i j}(n) s_{j}\right) t_{i n}\left(\lambda+\sum_{j=1}^{m} \varepsilon_{i j}(n)\left(1-s_{j}\right)\right),
\end{aligned}
$$

причем функция $h_{n}^{(i)}(\mathbf{s}):=\chi_{n}^{(i)}(\mathbf{s} ; 0)$ имеет вид

$$
h_{n}^{(i)}(\mathbf{s})=\left(\gamma_{i 0}(n)+\sum_{j=1}^{m} \gamma_{i j}(n) s_{j}\right) t_{i n}\left(\sum_{j=1}^{m} \varepsilon_{i j}(n)\left(1-s_{j}\right)\right) .
$$

П р и м е р 2 (ср. с [34]). Рассмотрим ту же систему поллинга, что и раньше, но предположим теперь, что на каждой станции прибор придерживается исчерпывающей дисциплины обслуживания с обратной связью: он обслуживает все требования, которые находились в очереди на станции в момент его прибытия, а также все требования, появившиеся на станции во время его визита, вплоть до момента, когда очередь требований на этой станции иссякнет, а затем мгновенно переходит на следующую станцию.

Покажем, что эта система также обладает свойством ветвления.

Поскольку во время $n$-го визита прибора на станцию $i$ каждое требование, находящееся на станции, обслуживается прибором случайное число раз, имеющее сдвинутое на 1 геометрическое распределение с параметром $\gamma_{i i}(n)$, то преобразование Лапласа $w_{i n}(\lambda)$ распределения случайной величины $\eta_{i}(n)$ - суммарного времени обслуживания требования на станции $i$ во время $n$-го цикла обслуживания, имеет вид

$$
w_{i n}(\lambda)=\frac{1-\gamma_{i i}(n)}{1-\gamma_{i i}(n) t_{i n}(\lambda)} t_{i n}(\lambda) .
$$

Обозначим $\left(\sigma_{i 1}(n), \ldots, \sigma_{i m}(n)\right)$ случайный вектор, имеющий то же распределение, что и вектор количества требований, прибывших на станции системы поллинга за суммарное время обслуживания такого требования в очереди $i$, которое после окончания обслуживания на станции $i$ переместилось либо на станцию $j \neq i$, либо покинуло систему. Положим

$$
y_{i n}(\mathbf{s}):=\frac{\gamma_{i 0}(n)+\sum_{j \neq i} \gamma_{i j}(n) s_{j}}{1-\gamma_{i i}(n)}
$$

Аналогично (1) можно показать, что

$$
\mathbf{E}\left[s_{i}^{\sigma_{i 1}(n)} \cdots s_{m}^{\sigma_{i m}(n)} e^{-\lambda \tau_{i}(n)}\right]=y_{i n}(\mathbf{s}) w_{i n}\left(\lambda+\sum_{j=1}^{m} \varepsilon_{i j}(n)\left(1-s_{j}\right)\right) .
$$

Пусть теперь $l_{\text {in }}(\lambda)$ - преобразование Лапласа распределения периода занятости $\eta_{i, \text { tot }}(n)$, порожденного отдельным требованием в системе 
обслуживания $M / G / 1$ с интенсивностью поступления требований $\varepsilon_{i i}(n)$ и распределением времени обслуживания таким же, как и у случайной величины $\eta_{i}(n)$, и пусть $\left(\theta_{i 1}(n), \ldots, \theta_{i m}(n)\right)$ - общее число новых требований, пришедших на $i$-ю станцию нашей системы поллинга в промежутке времени случайной длины, имеющем такое же распределение, как и $\eta_{i, \text { tot }}(n)$. В этом случае функция $l_{i n}(\lambda)$ является единственным решением уравнения

$$
l_{\text {in }}(\lambda)=w_{i n}\left(\lambda+\varepsilon_{i i}(n)\left(1-l_{\text {in }}(\lambda)\right)\right),
$$

a (cp. с [36]) функции

$$
\chi_{n}^{(i)}(\mathbf{s} ; \lambda):=\mathbf{E}\left[s_{1}^{\theta_{i 1}(n)} \cdots s_{m}^{\theta_{i m}(n)} e^{-\lambda \eta_{i, \mathrm{tot}}(n)}\right], \quad i=1, \ldots, m,
$$

являются (в наших условиях) единственными решениями уравнений

$$
\chi_{n}^{(i)}(\mathbf{s} ; \lambda)=y_{i n}(\mathbf{s}) w_{i n}\left(\lambda+\sum_{j \neq i} \varepsilon_{i j}(n)\left(1-s_{j}\right)+\varepsilon_{i i}(n)\left(1-\chi_{n}^{(i)}(\mathbf{s} ; \lambda)\right)\right) .
$$

В частности,

$$
h_{n}^{(i)}(\mathbf{s})=y_{i n}(\mathbf{s}) w_{i n}\left(\sum_{j \neq i} \varepsilon_{i j}(n)\left(1-s_{j}\right)+\varepsilon_{i i}(n)\left(1-h_{n}^{(i)}(\mathbf{s})\right)\right) .
$$

Заметим, что в рамках предложенного нами подхода можно также рассматривать модели, в которых требования поступают группами. В этом случае необходимо, например, всюду в $t_{i n}(\cdot)$ заменить переменные $s_{1}, \ldots, s_{m}$ соответствующими вероятностными производящими функциям размеров групп требований, поступающих на станции $j=1, \ldots, m$.

Поскольку мы требуем справедливость свойства ветвления для дисциплин обслуживания системы, то для читателя не будет сюрпризом тот факт, что при исследовании таких систем используются методы теории ветвящихся процессов. Такой подход не является новым. Системы поллинга, обладающие свойством ветвления, у которых производящие функции $h_{n}^{(i)}(\mathbf{s}), n=1,2, \ldots$, неслучайны и одни и те же для всех циклов, рассматривались в относительно ранних работах [24], [36], [42] и, совсем недавно, в статьях [9], [12] и [13]. Эти модели включают в себя многие классические дисциплины обслуживания, такие, как исчерпывающая, шлюзовая, биномиально-шлюзовая и их модификации с обратной связью (см. обзоры [4] и [35], где можно найти соответствующие определения и более детальное описание указанных моделей).

Системы поллинга, входные параметры и дисциплины обслуживания которых меняются случайным образом и (или) зависят от состояния системы, изучены не в столь большой степени общности. При анализе таких систем весьма часто используется либо метод жидкостной 
аппроксимации [21]-[23], либо метод, основанный на построении подходящих функций Ляпунова [32]-[34] (в последнем случае без ссылок на ветвящиеся процессы). Авторы статьи [34] пишут, что их модели можно обобщить. Однако «...это приведет к существенным усложнениям доказательств». В данной работе мы покажем, что редукция задач, связанных с системами поллинга ветвящегося типа с финальным продуктом, эволюционирующими в случайной среде, к соответствующим задачам для многотипных ветвящихся процессов с финальным продуктом, эволюционирующих в случайной среде, дает желаемые ответы при помощи единого метода и в общей ситуации. Поскольку в нашей модели с.в.р.ф. выбираются для каждого цикла независимо от прошлого, то мы допускаем различные системы обслуживания на разных станциях. Более того, дисциплины обслуживания на станциях могут меняться случайным образом от визита к визиту. В частности, мы допускаем чередование исчерпывающей и шлюзовой дисциплин. В настоящей работе мы рассматриваем лишь модели с мгновенными переключениями. Исследование систем поллинга с положительными (и случайными) временами переключений можно проводить сходными методами. Это, однако, требует больше усилий и будет сделано в другой статье.

Структура оставшейся части работы такова. Раздел 2 посвящен детальному описанию многотипных ветвящихся процессов с финальным продуктом, эволюционирующих в случайной среде. В разд. 3 мы напомним некоторые известные результаты для обычных многотипных ветвящихся процессов в случайной среде (ВПСС) и сформулируем основные результаты статьи, описывающие асимптотическое поведение совокупного объема финального продукта для докритических ВПСС. Раздел 4 содержит важное известное утверждение, связанное с асимптотическими свойствами хвостов распределений бесконечных сумм произведений случайных матриц. В разд. 5 получены некоторые оценки для моментов размеров популяций докритических ВПСС. Доказательства основных результатов работы собраны в разд. 7. И, наконец, в разд. 8 показано, как результаты, установленные для многотипных ветвящихся процессов с финальным продуктом, эволюционирующих в случайной среде, могут быть использованы для изучения вероятностных свойств различных характеристик систем поллинга ветвящегося типа.

\section{2. Ветвящиеся процессы в случайной среде с финальным}

продуктом. Как было сказано в предыдущем разделе, основной целью данной работы является анализ свойств периодов занятости и некоторых других характеристик систем поллинга ветвящегося типа методами теории ветвящихся процессов в случайной среде. Однако, прежде чем начать такой анализ, нам придется пройти относительно долгую дорогу обозначений и утверждений, чего практически невозможно избежать при 
исследовании многотипных ветвящихся процессов.

Пусть $\left(\xi_{1}, \ldots, \xi_{m} ; \varphi\right)-(m+1)$-мерный вектор, в котором компоненты $\xi_{1}, \ldots, \xi_{m}$ - неотрицательные целочисленные случайные величины, а $\varphi$-неотрицательная случайная величина. Обозначим

$$
F(\mathbf{s} ; \lambda)=\mathbf{E}\left[s_{1}^{\xi_{1}} \cdots s_{m}^{\xi_{m}} e^{-\lambda \varphi}\right], \quad \mathbf{s}=\left(s_{1}, \ldots, s_{m}\right) \in[0,1]^{m}, \quad \lambda \geqslant 0,
$$

соответствующую с.в.п.ф., и пусть $\mathscr{F}:=\{F(\mathbf{s} ; \lambda)\}-$ множество всех таких с.в.п.ф., а

$$
\mathscr{F}^{m}:=\left\{\mathbf{F}(\mathbf{s} ; \lambda)=\left(F^{(1)}(\mathbf{s} ; \lambda), \ldots, F^{(m)}(\mathbf{s} ; \lambda)\right): F^{(i)}(\mathbf{s} ; \lambda), i \in\{1, \ldots, m\}\right\}
$$

- $m$-кратное прямое произведение $\mathscr{F}$ с собой. Пусть, далее,

$$
\mathscr{F}_{0}:=\left\{f(\mathbf{s})=F(\mathbf{s} ; 0), \mathbf{s} \in[0,1]^{m}\right\}
$$

— множество всех обычных вероятностных производящих функций (в.п.ф.)

$$
f(\mathbf{s})=\mathbf{E}\left[s_{1}^{\xi_{1}} \cdots s_{m}^{\xi_{m}}\right]
$$

a

$$
\mathscr{F}_{0}^{m}:=\left\{\mathbf{f}(\mathbf{s})=\left(f^{(1)}(\mathbf{s}), \ldots, f^{(m)}(\mathbf{s})\right): f^{(i)}(\mathbf{s}), i \in\{1, \ldots, m\}\right\}
$$

- множество всех $m$-мерных (векторнозначных) в.п.ф. Будем предполагать, что на естественной $\sigma$-алгебре $\mathscr{A}$, порожденной подмножествами множества $\mathscr{F}_{\lambda}^{m}$, задана вероятностная мера $\mathbb{P}$. Пусть

$$
\mathbf{F}_{0}(\mathbf{s} ; \lambda), \mathbf{F}_{1}(\mathbf{s} ; \lambda), \ldots, \mathbf{F}_{k}(\mathbf{s} ; \lambda), \ldots \mathbf{c} \mathbf{F}_{n}(\mathbf{s} ; \lambda):=\left(F_{n}^{(1)}(\mathbf{s} ; \lambda), \ldots, F_{n}^{(m)}(\mathbf{s} ; \lambda)\right)
$$

- последовательность независимых векторнозначных с.в.п.ф., выбранных из $\mathscr{F}^{m}$ в соответствии с мерой $\mathbb{P}$. Последовательность $\left\{\mathbf{F}_{n}(\mathbf{s} ; \lambda), n \in\right.$ $\left.\mathbb{N}_{0}\right\}$ называется случайной средой. Свяжем со с.в.п.ф. $F_{n}^{(i)}(\mathbf{s} ; \lambda)$ случайный вектор непосредственных потомков $\boldsymbol{\xi}_{i}(n):=\left(\xi_{i 1}(n), \ldots, \xi_{i m}(n)\right)$ и случайную величину $\varphi_{i}(n)$ такие, что

$$
F_{n}^{(i)}(\mathbf{s} ; \lambda)=\mathbf{E}\left[s_{1}^{\xi_{i 1}(n)} \cdots s_{m}^{\xi_{i m}(n)} e^{-\lambda \varphi_{i}(n)}\right]
$$

Теперь мы можем дать неформальное описание многотипного ветвящегося процесса с финальным продуктом, эволюционирующего в случайной среде

$$
\mathbf{R}(n)=(\mathbf{Z}(n) ; \Phi(n)), \quad n \in \mathbb{N}_{0},
$$

который для простоты мы здесь называем просто $(m, \varphi)$-процессом. Этот процесс можно рассматривать как процесс развития популяции, состоящей из $m$ типов частиц, в которой накапливается финальный продукт. 
Начальными условиями процесса являются: вектор числа частиц $\mathbf{Z}(0)=\left(Z_{1}(0), \ldots, Z_{m}(0)\right)$ (возможно, случайный), где $Z_{i}(0)-$ число частиц типа $i \in\{1, \ldots, m\}$ в процессе в момент 0 , и финальный продукт (возможно, случайного) объема $\Phi(0)$. Все первоначальные частицы имеют единичную продолжительность жизни и в момент гибели производят потомков и финальный продукт независимо друг от друга. Например, частица, скажем, типа $i$ производит потомков различных типов и финальный продукт, добавляемый к уже накопленному в популяции, в соответствии со с.в.п.ф. $F_{0}^{(i)}(\mathbf{s} ; \lambda)$. Новорожденные частицы образуют первое поколение $(m, \varphi)$-процесса, имеют единичную продолжительность жизни и, погибая, производят, независимо друг от друга потомков и финальный продукт в соответствии со своими типами и согласно со с.в.п.ф. $F_{1}^{(i)}(\mathbf{s} ; \lambda), i=1,2, \ldots, m$, и так далее.

Точное определение интересующего нас процесса выглядит следующим образом.

О п р е д е л е н и е $1 .(m+1)$-мерным ветвящимся процессом Гальтона-Ватсона

$$
\mathbf{R}_{\varphi}(n)=\mathbf{R}(n):=(\mathbf{Z}(n) ; \Phi(n))=\left(Z_{1}(n), \ldots, Z_{m}(n) ; \Phi(n)\right), \quad n \in \mathbb{N}_{0},
$$

с финальным продуктом $\varphi$ в фиксированной (но выбранной случайно) среде $\left\{\mathbf{F}_{n}(\mathbf{s} ; \lambda), n \geqslant 0\right\}$ или просто $(m, \varphi)$-процессом называется неоднородный марковский процесс с пространством состояний

$$
\mathbb{N}_{0}^{m} \times \mathbb{R}_{0}:=\left\{z=\left(z_{1}, \ldots, z_{m} ; w\right), z_{i} \in \mathbb{N}_{0} ; w \in[0, \infty)\right\}
$$

задаваемый соотношениями

$$
\begin{gathered}
\mathbf{R}(0)=(\mathbf{Z}(0) ; \Phi(0)), \\
\mathbf{E}\left[\mathbf{s}^{\mathbf{Z}(n+1)} e^{-\lambda \Phi(n+1)} \mid \mathbf{F}_{0}, \ldots, \mathbf{F}_{n} ; \mathbf{R}(0), \ldots, \mathbf{R}(n)\right]=e^{-\lambda \Phi(n)}\left(\mathbf{F}_{n}(\mathbf{s} ; \lambda)\right)^{\mathbf{Z}(n)}
\end{gathered}
$$

Заметим, что начальное значение $\mathbf{R}(0)$ может быть случайным (что каждый раз будет оговариваться дополнительно) и, по причинам, связанным с применениями к системам обслуживания, мы не исключаем случай $\mathbf{z}=\mathbf{0}$.

В дальнейшем, с целью упрощения обозначений, мы полагаем

$$
\mathbf{E}_{\mathbf{F}}\left[\mathbf{s}^{\mathbf{Z}(n+1)} e^{-\lambda \Phi(n+1)}\right]:=\mathbf{E}\left[\mathbf{s}^{\mathbf{Z}(n+1)} e^{-\lambda \Phi(n+1)} \mid \mathbf{F}_{0}, \ldots, \mathbf{F}_{n}\right] .
$$

Таким образом,

$$
\mathbf{E}_{\mathbf{F}}\left[\mathbf{s}^{\mathbf{Z}(n+1)} e^{-\lambda \Phi(n+1)} \mid \mathbf{R}(0), \ldots, \mathbf{R}(n)\right]=e^{-\lambda \Phi(n)}\left(\mathbf{F}_{n}(\mathbf{s} ; \lambda)\right)^{\mathbf{Z}(n)},
$$


или

$$
\begin{aligned}
& \mathbf{R}(n+1)=(\mathbf{0} ; \Phi(n))+\sum_{i=1}^{m} \sum_{k=1}^{Z_{i}(n)}\left(\boldsymbol{\xi}_{i}(n ; k) ; \varphi_{i}(n ; k)\right) \\
& \Phi(n+1)=\Phi(0)+\sum_{l=0}^{n} \sum_{i=1}^{m} \sum_{k=1}^{Z_{i}(l)} \varphi_{i}(l ; k)
\end{aligned}
$$

где случайный вектор $\left(\boldsymbol{\xi}_{i}(n ; k) ; \varphi_{i}(n ; k)\right)$ представляет число потомков и финальный продукт $k$-й частицы типа $i$ в $n$-м поколении процесса, причем при фиксации среды и каждых $n=0,1, \ldots$ и $i \in\{1, \ldots, m\}$ векторы

$$
\left(\boldsymbol{\xi}_{i}(n ; k) ; \varphi_{i}(n ; k)\right), \quad k=1,2, \ldots, Z_{i}(n),
$$

независимы и одинаково распределены:

$$
\left(\boldsymbol{\xi}_{i}(n ; k) ; \varphi_{i}(n ; k)\right) \stackrel{d}{=}\left(\boldsymbol{\xi}_{i}(n) ; \varphi_{i}(n)\right) .
$$

Заметим, что если $\Phi(0)=0$ и $\varphi_{i}(n ; k) \equiv 1$, то величина $\Phi(n)$ равна общему числу частиц, родившихся в процессе в поколениях $0,1, \ldots, n-1$; если же $\Phi(0)=0$, а $\varphi_{i}(n ; k)=I\left\{\sum_{j=1}^{m} \xi_{i j}(n ; k) \geqslant t\right\}$ для некоторого натурального $t$ (здесь и в дальнейшем $I\{A\}$ обозначает индикатор события $A)$, то величина $\Phi(N)$ равна общему числу таких частиц всех типов в поколениях $0,1, \ldots, N-1$, каждая из которых имела по крайней мере $t$ прямых потомков, и так далее.

Полагая $\lambda=0$, мы приходим к определению обычного многотипного ветвящегося процесса в случайной среде (ВПСС), который мы будем называть подчиненныл ВПСС для исходного $(m, \varphi)$-процесса.

О п р е д е л е н и е 2. Процессом Гальтона-Ватсона

$$
\mathbf{Z}(n)=\left(Z_{1}(n), \ldots, Z_{m}(n)\right), \quad n \in \mathbb{N}_{0},
$$

с $m$ типами частиц в фиксированной (но выбранной случайно) среде $\left\{\mathbf{f}_{n}(\mathbf{s}), n \geqslant 0\right\}$ называется неоднородный марковский процесс с пространством состояний такой, что

$$
\mathbf{Z}(0)=\mathbf{z}, \quad \mathbf{E}\left[\mathbf{s}^{\mathbf{Z}(n+1)} \mid \mathbf{f}_{0}, \ldots, \mathbf{f}_{n} ; \mathbf{Z}(0), \ldots, \mathbf{Z}(n)\right]=\left(\mathbf{f}_{n}(\mathbf{s})\right)^{\mathbf{Z}(n)}
$$

Для упрощения обозначений будем писать

$$
\mathbf{E}_{\mathbf{f}}\left[\mathbf{s}^{\mathbf{Z}(n+1)}\right]:=\mathbf{E}\left[\mathbf{s}^{\mathbf{Z}(n+1)} \mid \mathbf{f}_{0}, \ldots, \mathbf{f}_{n}\right]
$$

Из (8) вытекает, что

$$
\mathbf{Z}(n+1):=\sum_{i=1}^{m} \sum_{k=1}^{Z_{i}(n)} \boldsymbol{\xi}_{i}(n ; k),
$$


где $\boldsymbol{\xi}_{i}(n ; k) \stackrel{d}{=} \boldsymbol{\xi}_{i}(n), k=1, \ldots, Z_{i}(n)$, и, при фиксации среды и каждых $n=0,1, \ldots$ и $i \in\{1, \ldots, m\}$, указанные векторы независимы.

Обозначим

$$
A_{n}=\left(a_{i j}(n)\right)_{i, j=1}^{m}:=\left(\left.\frac{\partial F_{n}^{(i)}(\mathbf{s}, \lambda)}{\partial s_{j}}\right|_{\mathbf{s}=\mathbf{1}, \lambda=0}\right)_{i, j=1}^{m}=\left(\left.\frac{\partial f_{n}^{(i)}(\mathbf{s})}{\partial s_{j}}\right|_{\mathbf{s}=\mathbf{1}}\right)_{i, j=1}^{m}
$$

матрицу средних векторнозначной в.п.ф. $\mathbf{f}_{n}$ и положим

$$
\begin{aligned}
\mathbf{C}_{n} & :=\left(\mathbf{E}_{\mathbf{F}} \varphi_{1}(n), \ldots, \mathbf{E}_{\mathbf{F}} \varphi_{1}(n)\right)^{\prime} \\
& =\left(\left.\frac{\partial F_{n}^{(1)}(\mathbf{s}, \lambda)}{\partial \lambda}\right|_{\mathbf{s}=\mathbf{1}, \lambda=0}, \ldots,\left.\frac{\partial F_{n}^{(m)}(\mathbf{s}, \lambda)}{\partial \lambda}\right|_{\mathbf{s}=\mathbf{1}, \lambda=0}\right)^{\prime} .
\end{aligned}
$$

В силу наших допущений пары $\left(A_{n}, \mathbf{C}_{n}\right), n=0,1, \ldots$, независимы и одинаково распределены: $\left(A_{n}, \mathbf{C}_{n}\right) \stackrel{d}{=}(A, \mathbf{C})$. Предположим, что

$$
\mathbf{E} \ln ^{+}\|A\|<\infty \text {. }
$$

Известно (см., например, [31]), что если выполнено условие (11), то предел

$$
\lim _{n \rightarrow \infty} \frac{1}{n} \ln \left\|A_{n-1} A_{n-2} \cdots A_{0}\right\|=: \alpha
$$

существует с вероятностью 1 и, более того,

$$
\lim _{n \rightarrow \infty} \frac{1}{n} \mathbf{E} \ln \left\|A_{n-1} A_{n-2} \cdots A_{0}\right\|=\alpha .
$$

В дальнейшем будем называть ВПСС докритическим, если $\alpha<0$, и надкритическим, если $\alpha>0$.

Напомним, что ВПСС с одним типом частиц и независимыми одинаково распределенными в.п.ф. числа потомков ввели У.Смит и У. Вилкинсон в работе [37]. Более общие модели процессов с одним типом частиц рассматривались в статьях [14]-[16]. Процессы указанного типа изучались многими авторами (см. обзор [39], где имеются ссылки на работы, опубликованные до 1985 г., а также статьи [1]-[3], [7], [10], [11], [20], [25], [40] и [41], содержащие более поздние результаты). ВПСС исследовались, в частности, в работах [14], [30] и [38].

Обычные процессы Гальтона-Ватсона с одним типом частиц и финальным продуктом рассматривали Б. А. Севастьянов [8] (в случае целочисленных неотрицательных значений $\varphi(n ; k))$ и С. А. Гришечкин [6] (для произвольных неотрицательных значений $\varphi(n ; k)$ ). Гришечкин использовал процессы Гальтона-Ватсона и марковские ветвящиеся процессы с непрерывным временем и финальным продуктом (с иммиграцией и без нее) при исследовании систем обслуживания с разделением процессора [5]. 
3. Предельные теоремы для $(m, \varphi)$-процессов. Обозначим

$$
\Pi_{l, n}:=\prod_{i=l}^{n-1} A_{i}, \quad 1 \leqslant l \leqslant n,
$$

полагая, что $\Pi_{n, n}:=E$ является единичной матрицей размера $m \times m$. Для векторов $\mathbf{u}=\left(u_{1}, \ldots, u_{m}\right), \mathbf{v}=\left(v_{1}, \ldots, v_{m}\right)^{\prime} \in \mathbb{R}^{m}$ обозначим

$$
\langle\mathbf{u}, \mathbf{v}\rangle:=\sum_{k=1}^{m} u_{i} v_{i}
$$

их скалярное произведение.

Для матрицы $X=\left(x_{i j}\right)_{i, j=1}^{m}$ размера $m \times m$ и $m$-мерного вектора $\mathbf{u}=\left(u_{1}, \ldots, u_{m}\right)$ введем нормы

$$
\|X\|:=\sum_{i, j=1}^{m}\left|x_{i j}\right|, \quad\|\mathbf{u}\|:=\sum_{i=1}^{m}\left|u_{i}\right|
$$

и

$$
\|X\|_{2}:=\sqrt{\sum_{i, j=1}^{m}\left|x_{i j}\right|^{2}}, \quad\|\mathbf{u}\|_{2}:=\sqrt{\sum_{i=1}^{m}\left|u_{i}\right|^{2}} .
$$

Сформулируем теперь важное утверждение, касающееся свойств ВПСС.

Пусть

$$
q_{i}(\mathbf{f}):=\lim _{n \rightarrow \infty} \mathbf{P}_{\mathbf{f}}\left(\|\mathbf{Z}(n)\|=0 \mid \mathbf{Z}(0)=\mathbf{e}_{i}\right), \quad i=1, \ldots, m,
$$

- вероятность вырождения МВПСС, порожденного в момент 0 одной частицей типа $i$, и

$$
\mathbf{q}(\mathbf{f}):=\left(q_{1}(\mathbf{f}), \ldots, q_{m}(\mathbf{f})\right) .
$$

Теорема 1 [38]. Если матриџы средних ВПСС удовлетворяют условию (11) и найдутся натуральное число L такое, что

$$
\mathbf{P}\left(\min _{1 \leqslant i, j \leqslant m}\left(A_{L-1} A_{L-2} \cdots A_{0}\right)_{i j}>0\right)=1,
$$

и число $1 \leqslant l \leqslant m$ такое, ито

$$
\mathbf{E}\left|\ln \left(1-\mathbf{P}_{\mathbf{f}}\left(\mathbf{Z}_{l}(L)=0 \mid \mathbf{Z}(0)=\mathbf{e}_{i}\right)\right)\right|<\infty,
$$

то для параметра $\alpha$, определенного в (13), выполнено следующее:

1) если $\alpha<0$, то $\mathbf{P}(\mathbf{q}(\mathbf{f})=\mathbf{1})=1$

2) если $\alpha>0$, по $\mathbf{P}(\mathbf{q}(\mathbf{f})<\mathbf{1})=1 u$

$$
\mathbf{P}_{\mathbf{f}}\left(\lim _{n \rightarrow \infty} n^{-1} \ln \|\mathbf{Z}(n)\|=\alpha \mid \mathbf{Z}(0)=\mathbf{e}_{i}\right)=1-q_{i}(\mathbf{f})
$$

с вероятностью 1 для $1 \leqslant i \leqslant m$. 
Пусть $\tau$ - момент вырождения ВПСС, стартующего с вектора числа частиц $\mathbf{Z}(0)$ (может быть, случайного) такого, что $\mathbf{E}\|\mathbf{Z}(0)\|<\infty$. Ясно, что,

$$
\begin{aligned}
\mathbf{P}(\tau>n) & =\mathbf{P}(\|\mathbf{Z}(n)\| \geqslant 1) \leqslant \mathbf{E}\|\mathbf{Z}(n)\|=\mathbf{E}\left\|\mathbf{Z}(0) A_{0} A_{1} \cdots A_{n-1}\right\| \\
& \leqslant \mathbf{E}\|\mathbf{Z}(0)\| \mathbf{E}\left\|A_{0} A_{1} \cdots A_{n-1}\right\| .
\end{aligned}
$$

Отсюда следует, что если $\alpha<0$, то для любого $\alpha_{*} \in(0,-\alpha)$ найдется константа $K_{*}=K_{*}\left(\alpha_{*}\right) \in(0, \infty)$ такая, что для любого $n=0,1,2, \ldots$

$$
\mathbf{P}(\tau>n) \leqslant K_{*} e^{-\alpha_{*} n} .
$$

Пусть

$$
\Phi:=\lim _{n \rightarrow \infty} \Phi(n)=\Phi(0)+\sum_{n=0}^{\infty} \sum_{i=1}^{m} \sum_{k=1}^{Z_{i}(n)} \varphi_{i}(n ; k)
$$

- совокупный финальный продукт, произведенный частицами $(m, \varphi)$ процесса до момента вырождения (если такое событие произошло). Легко видеть, что если подчиненный ВПСС - надкритический, удовлетворяет условиям теоремы 1 и, кроме того, финальный продукт $(m, \varphi)$ процесса подчиняется ограничению

$$
\mathbf{P}\left(\min _{1 \leqslant l \leqslant m} \mathbf{E}_{\mathbf{F}} \varphi_{l}>0\right)>0,
$$

то в силу (14) и закона больших чисел для любого $i=1, \ldots, m$

$$
\begin{aligned}
\mathbf{P}(\Phi & \left.=\infty \mid \mathbf{Z}(0)=\mathbf{e}_{i}\right) \\
& \geqslant \mathbf{P}\left(\liminf _{n \rightarrow \infty}\left(\sum_{j=1}^{m} \sum_{k=1}^{Z_{j}(n)} \varphi_{j}(n ; k)\right)=\infty \mid \mathbf{Z}(0)=\mathbf{e}_{i}\right)>0 .
\end{aligned}
$$

Заметим, наконец, что если $\Phi(0)=0$ и $\varphi_{j}(n ; k) \equiv 1$, то величина

$$
\Phi:=\lim _{n \rightarrow \infty} \Phi(n)=\sum_{n=0}^{\infty}\|\mathbf{Z}(n)\|
$$

равна общему числу частиц, существовавших в $(m, \varphi)$-процессе, или, что одно и то же, в подчиненном ВПСС за время его эволюции.

Для $x \geqslant 0$ положим

$$
s(x):=\lim _{n \rightarrow \infty}\left(\mathbf{E}\left\|A_{n-1} \cdots A_{0}\right\|^{x}\right)^{1 / n}=\lim _{n \rightarrow \infty}\left(\mathbf{E}\left\|\Pi_{0, n}\right\|^{x}\right)^{1 / n},
$$

и пусть

$$
s^{\prime}(0)=\lim _{n \rightarrow \infty} \frac{1}{n} \mathbf{E} \ln \left\|A_{n-1} \cdots A_{0}\right\|=\lim _{n \rightarrow \infty} \frac{1}{n} \mathbf{E} \ln \left\|\Pi_{0, n}\right\|
$$


обозначает верхний показатель Ляпунова для данной последовательности матриц.

Введем множество $\mathscr{D}:=\left\{x>0: \mathbf{E}\left\|A_{0}\right\|^{x}<\infty\right\}$. Известно, что пределы в (18) и (19) существуют и, более того, функция $s(x)$ является логарифмически выпуклой на множестве $\mathscr{D}$ (см., например, [31]). Положим

$$
\kappa:=\inf \{x>0: s(x)>1\},
$$

считая, что $\kappa=\infty$, если $s(x) \leqslant 1$ для всех $x>0$. Заметим, что $s(0)=1$, и, следовательно, $\kappa=0$, если $s^{\prime}(0)>0$, и $\kappa \in(0, \infty]$, если $s^{\prime}(0)<0$.

В последнем случае (которому будет уделено основное внимание) ряд $\sum_{n=0}^{\infty} \mathbf{E}\left\|\Pi_{0, n}\right\|^{x}$ сходится при $0<x<\kappa$ и расходится при $x>\kappa$.

Введем множество

$$
U_{+}=\left\{\mathbf{u}=\left(u_{1}, \ldots, u_{m}\right) \in \mathbb{R}^{m}: u_{i} \geqslant 0,1 \leqslant i \leqslant m,\|\mathbf{u}\|_{2}=1\right\}
$$

и свяжем с набором $\left(A_{n}, \mathbf{C}_{n}\right), n=0,1,2, \ldots$, независимых одинаково распределенных пар ряды

$$
\Xi_{l}:=\sum_{k=l}^{\infty} A_{l} A_{l+1} \cdots A_{k-1} \mathbf{C}_{k}=\sum_{k=l}^{\infty} \Pi_{l, k} \mathbf{C}_{k}, \quad l=0,1, \ldots ; \quad \Xi=: \Xi_{0} .
$$

Наши основные результаты доказаны при выполнении следующего условия.

У с л о в и е Т. Существуют константа $K_{0}$ и непрерывная строго положительная функция $l(\mathbf{u})$ на $U_{+}$такие, что для всех $\mathbf{u} \in U_{+}$

$$
\lim _{y \rightarrow \infty} y^{\kappa} \mathbf{P}(\langle\mathbf{u}, \Xi\rangle>y)=K_{0} l(\mathbf{u}) .
$$

В разд. 4 мы укажем достаточные условия на распределение пар $\left(A_{n}, \mathbf{C}_{n}\right)$, обеспечивающие выполнение условия $\mathrm{T}$. Эти условия заимствованы из работы [28], в которой исследовались свойства хвостов распределений сумм и произведений случайных матриц.

Следующая теорема является основным результатом статьи.

Теорема 2. Пусть $(m, \varphi)$-прочесс удовлетворяет следуюшим предположениям:

1) подчиненный ВПСС является докритическим и удовлетворяет условиям теоремь 1 ;

2) для параметра к, определенного в (20), выполнены следующие условия:

$$
\begin{aligned}
& \text { если } \kappa>1 \text {, то } \\
& \max _{1 \leqslant i \leqslant m} \mathbf{E}\left|\sum_{j=1}^{m}\left(\xi_{i j}-a_{i j}\right)\right|^{\kappa}<\infty \quad u \quad \mathbf{E}\left|\sum_{i=1}^{m}\left(\varphi_{i}-\mathbf{E}_{\mathbf{F}} \varphi_{i}\right)\right|^{\kappa}<\infty,
\end{aligned}
$$


если $\kappa \leqslant 1, m o$

$$
\max _{1 \leqslant i \leqslant m} \mathbf{E}\left(\sum_{j=1}^{m} \mathbf{D}_{\mathbf{F}} \xi_{i j}\right)^{\kappa}<\infty \quad u \quad \mathbf{E}\left(\sum_{i=1}^{m} \mathbf{D}_{\mathbf{F}} \varphi_{i}\right)^{\kappa}<\infty
$$

3) найдется $\delta>0$ такое, ито $0<\mathbf{E} \varphi_{i}^{\kappa+\delta}<\infty, i=1, \ldots, m$.

Если, кроме того, матрица средних (9) и вектор (10) таковь, что выполнено условие $\mathrm{T}$, то при $y \rightarrow \infty$

$$
\mathbf{P}(\Phi>y) \sim C y^{-\kappa}, \quad C \in(0, \infty) .
$$

Очевидным следствием теоремы 2 является следующее утверждение.

Теорема 3. Если выполнень условия теоремь 2, то

$$
\mathbf{E} \Phi^{x}<\infty
$$

тогда и только тогда, когда $x<\kappa$.

4. Вспомогательные результаты. Доказательство теоремы 2 существенным образом использует условие Т, выполнение которого проверить непросто. Мы перечислим здесь множество условий, указанных в [28], совокупность которых обеспечивает справедливость условия T.

Пусть $\Lambda(A)$ - спектральный радиус матрицы $A$. Следующее утверждение является уточнением теоремы Кестена из работы [28].

Теорема 4 (см. [18]). Пусть $\left\{A_{n}, n \geqslant 0\right\}$ - последовательность независимых одинаково распределенных матрии, порожденных мерой $\mathbb{P}_{A}$, с носителем на множестве матрии с неотрицательными элементами, и пусть $A=\left(a_{i j}\right)_{i, j=1}^{m} \stackrel{d}{=} A_{n}$. Предположим, что выполнены следуюшие условия:

1) существует иисло $\varepsilon>0$ такое, что $\mathbf{E}\|A\|^{\varepsilon}<\infty$;

2) матрица $A$ почти наверное не имеет нулевых столбцов;

3) группа, порожденная набором чисел

$\left\{\ln \Lambda\left(a_{n} \cdots a_{0}\right): a_{n} \cdots a_{0}>0\right.$ для некоторого $n$ и матрии $\left.a_{i} \in \operatorname{supp}\left(\mathbb{P}_{A}\right)\right\}$ плотна в $\mathbb{R}$;

4) существует число $\kappa_{0}>0$ такое, что

$$
\mathbf{E}\left[\min _{1 \leqslant i \leqslant m}\left(\sum_{j=1}^{m} a_{i j}\right)^{\kappa_{0}}\right] \geqslant m^{\kappa_{0} / 2} \quad u \quad \mathbf{E}\|A\|^{\kappa_{0}} \ln ^{+}\|A\|<\infty .
$$

Тогда найдется число $\kappa \in\left(0, \kappa_{0}\right]$ такое, что

$$
s^{\prime}(\kappa)=\lim _{n \rightarrow \infty} \frac{1}{n} \ln \mathbf{E}\left\|A_{n-1} \cdots A_{0}\right\|^{\kappa}=0 .
$$


Если, кроме того, набор т-мерных векторов $\left\{\mathbf{C}_{n}, n \geqslant 0\right\}$ таков, ито пары $\left(A_{n}, \mathbf{C}_{n}\right), n=0,1, \ldots$, независимь и одинаково распределень: $\left(A_{n}, \mathbf{C}_{n}\right) \stackrel{d}{=}(A, \mathbf{C})$, причем

$$
\mathbf{P}(\mathbf{C}=\mathbf{0})<1, \quad \mathbf{P}(\mathbf{C} \geqslant \mathbf{0})=1, \quad \mathbf{E}\|\mathbf{C}\|^{\kappa}<\infty,
$$

то найдутся константа $K_{0} \in(0, \infty)$ и непрерьвная строго положительная на $U_{+}$функция $l(\mathbf{u})$ такие, что

$$
\lim _{y \rightarrow \infty} y^{\kappa} \mathbf{P}(\langle\mathbf{u}, \Xi\rangle>y)=K_{0} l(\mathbf{u}), \quad \mathbf{u} \in U_{+} .
$$

Следующая лемма имеет важное значение для дальнейших рассуждений.

Лемма 1 [27, теорема 1.5.1]. Если $X_{i}, i=1,2, \ldots$, - последовательность независимьх одинаково распределенньх случайньх величин таких, что $\mathbf{E}\left|X_{i}\right|^{p}<\infty u \mathbf{E} X_{i}=0$ при $p \geqslant 1$, а $N$ - момент остановки для последовательности $\Gamma_{n}:=X_{1}+\cdots+X_{n}$, то найдется константа $R_{p} \in(0, \infty)$ такая, ито

$$
\mathbf{E}\left|\Gamma_{N}\right|^{p} \leqslant R_{p} \mathbf{E}\left|X_{i}\right|^{p} \mathbf{E} N^{p / 2 \vee 1} .
$$

5. Свойства подчиненного ВПСС. Всюду в этом пункте предполагается, что условия теоремы 2 выполнены. Это, в частности, означает, что мы будем рассматривать докритические ВПСС. Условимся в дальнейшем обозначать символами $K, K_{x}, x \in(0, \infty)$, положительные константы, которые могут быть различными в различных формулах.

Оценим сначала сверху математическое ожидание случайной величины $\|\mathbf{Z}(n)\|^{x}, 0<x<\kappa$.

Лемма 2. Если $\mathbf{Z}(0)=\mathbf{z} u \kappa>1$, то для любого $x \in[1, \kappa)$ существуют числа $\rho_{x} \in(0,1)$ и $K_{x}<\infty$ такие, что

$$
\mathbf{E}\|\mathbf{Z}(n)\|^{x} \leqslant K_{x} \rho_{x}^{n}\|\mathbf{Z}\|^{x}
$$

для всех $n=1,2, \ldots$.

Д о к а з а т е л ь с т в о. Ясно, что для любого неслучайного вектора $\mathbf{b} \in \mathbb{R}^{m}$

$$
\begin{aligned}
\mathbf{E}\langle\mathbf{Z}(n), \mathbf{b}\rangle & =\mathbf{E}\left\langle\sum_{i=1}^{m} \sum_{k=1}^{Z_{i}(n-1)} \boldsymbol{\xi}_{i}(n-1 ; k), \mathbf{b}\right\rangle \\
& =\mathbf{E}\left\langle\mathbf{Z}(n-1), A_{n-1} \mathbf{b}\right\rangle=\mathbf{E}\left\langle\mathbf{Z}(n-j), \Pi_{n-j, n} \mathbf{b}\right\rangle \\
& =\cdots=\mathbf{E}\left\langle\mathbf{z}, \Pi_{0, n} \mathbf{b}\right\rangle, \quad 0 \leqslant j \leqslant n .
\end{aligned}
$$


Таким образом, если $\|\mathbf{b}\| \leqslant K<\infty$, то для любого $\delta>0$ такого, что $s(1)(1+\delta)<1$, найдется константа $K_{1} \in(0, \infty)$ такая, что для всех $n=1,2, \ldots$ и $0 \leqslant j \leqslant n$ справедлива оценка

$$
\left|\mathbf{E}\left\langle\mathbf{Z}(n-j), \Pi_{n-j, n} \mathbf{b}\right\rangle\right| \leqslant\|\mathbf{z}\| \mathbf{E}\left\|\Pi_{0, n}\right\| \mathbf{E}\|\mathbf{b}\| \leqslant K_{1}(s(1)(1+\delta))^{n}\|\mathbf{z}\| .
$$

Применим теперь рассуждения, сходные с использованными в работе [19]. Легко проверить, что для любых чисел $y, w \geqslant 0$ и любого $\varepsilon \in(0,1)$ выполнено соотношение

$$
y^{x} \leqslant(1+\varepsilon) w^{x}+c_{x, \varepsilon}|y-w|^{x},
$$

где $c_{x, \varepsilon}:=\left(1-(1+\varepsilon)^{-1 / x}\right)^{-x}$. Отсюда

$$
\begin{aligned}
\mathbf{E}\|\mathbf{Z}(n)\|^{x} & =\mathbf{E}\left\|\mathbf{Z}(n-1) A_{n-1}+\mathbf{Z}(n)-\mathbf{Z}(n-1) A_{n-1}\right\|^{x} \\
& \leqslant(1+\varepsilon) \mathbf{E}\left\|\mathbf{Z}(n-1) A_{n-1}\right\|^{x}+c_{x, \varepsilon} \mathbf{E}\left\|\mathbf{Z}(n)-\mathbf{Z}(n-1) A_{n-1}\right\|^{x} .
\end{aligned}
$$

Отталкиваясь от определения $a_{i j}(n)=\mathbf{E}_{\mathbf{F}} \xi_{i j}(n)$, положим

$$
\beta_{i}(n):=\sum_{j=1}^{m}\left(\boldsymbol{\xi}_{i j}(n)-a_{i j}(n)\right)
$$

и обозначим

$$
M_{x}(n ; i):=\mathbf{E}_{\mathbf{F}}\left|\beta_{i}(n)\right|^{x}, \quad M_{x}:=\max _{1 \leqslant i \leqslant m} \mathbf{E}\left|\beta_{i}(n)\right|^{x} .
$$

Используя лемму 1 при $p=x>1$, заключаем, что

$$
\begin{aligned}
& \mathbf{E}_{\mathbf{F}}\left\|\mathbf{Z}(n)-\mathbf{Z}(n-1) A_{n-1}\right\|^{x} \\
& \quad=\mathbf{E}_{\mathbf{F}}\left|\sum_{i=1}^{m} \sum_{k=1}^{Z_{i}(n-1)} \sum_{j=1}^{m}\left[\boldsymbol{\xi}_{i j}(n-1 ; k)-a_{i j}(n-1)\right]\right|^{x} \\
& \quad \leqslant m^{x} \sum_{i=1}^{m} \mathbf{E}_{\mathbf{F}}\left|\sum_{k=1}^{Z_{i}(n-1)} \sum_{j=1}^{m}\left[\boldsymbol{\xi}_{i j}(n-1 ; k)-a_{i j}(n-1)\right]\right|^{x} \\
& \quad \leqslant R_{x} m^{x} \sum_{i=1}^{m} M_{x}(n-1, i) \mathbf{E}_{\mathbf{F}} Z_{i}^{x / 2 \vee 1}(n-1) .
\end{aligned}
$$

Эти оценки и (25) дают

$$
\begin{aligned}
\mathbf{E}\|\mathbf{Z}(n)\|^{x} \leqslant & (1+\varepsilon) \mathbf{E}\left\|\mathbf{Z}(n-1) A_{n-1}\right\|^{x} \\
& +c_{x, \varepsilon} R_{x} m^{x} M_{x} \sum_{i=1}^{m} \mathbf{E} Z_{i}^{x / 2 \vee 1}(n-1) .
\end{aligned}
$$


Теперь мы готовы продемонстрировать справедливость оценки (23). Докажем сначала соотношение (23) для всех целых значений $x \in[1, \kappa)$. Для $x=1$ неравенство (23) вытекает из (24) при $\mathbf{b}=\mathbf{1}$. Воспользуемся теперь индукцией по $x \geqslant 2$. Замечая, что в этом случае $x / 2 \vee 1 \leqslant x-1$, мы видим при помощи (28) и оценки

$$
\sum_{i=1}^{m} \mathbf{E} Z_{i}^{x / 2 \vee 1}(n-1) \leqslant \mathbf{E} \sum_{i=1}^{m} Z_{i}^{x-1}(n-1) \leqslant \mathbf{E}\|\mathbf{Z}(n-1)\|^{x-1},
$$

что

$$
\begin{aligned}
\mathbf{E}\|\mathbf{Z}(n)\|^{x} \leqslant & (1+\varepsilon) \mathbf{E}\left\|\mathbf{Z}(n-1) A_{n-1}\right\|^{x}+c_{x, \varepsilon} R_{x} m^{x} M_{x} \mathbf{E}\|\mathbf{Z}(n-1)\|^{x-1} \\
\leqslant & (1+\varepsilon)^{2} \mathbf{E}\left\|\mathbf{Z}(n-2) A_{n-2} A_{n-1}\right\|^{x} \\
& +(1+\varepsilon) c_{x, \varepsilon} R_{x} m^{x} M_{x} \mathbf{E}\left\|A_{n-1}\right\|^{x} \mathbf{E}\|\mathbf{Z}(n-2)\|^{x-1} \\
& +c_{x, \varepsilon} R_{x} m^{x} M_{x} \mathbf{E}\|\mathbf{Z}(n-1)\|^{x-1} \leqslant \cdots \\
\leqslant & (1+\varepsilon)^{n} \mathbf{E}\left\|\mathbf{z} \Pi_{0, n}\right\|^{x}+c_{x, \varepsilon} R_{x} m^{x} M_{x} \\
& \times \sum_{j=0}^{n-1}(1+\varepsilon)^{j} \mathbf{E}\left\|\Pi_{n-j, n}\right\|^{x} \mathbf{E}\|\mathbf{Z}(n-j-1)\|^{x-1}
\end{aligned}
$$

Поскольку $x \in[1, \kappa)$, то для любого $\delta>0$ найдется константа $L_{x}$ такая, что

$$
\mathbf{E}\left\|\Pi_{0, n}\right\|^{x} \leqslant L_{x}(s(x)(1+\delta))^{n}
$$

при всех $n=0,1,2, \ldots$. По предположению индукции существуют константы $K_{x-1}$ и $\rho_{x-1} \in(s(x), 1)$ такие, что

$$
\mathbf{E}\|\mathbf{Z}(n-j)\|^{x-1} \leqslant K_{x-1} \rho_{x-1}^{n-j}\|\mathbf{z}\|^{x-1} \leqslant K_{x-1} \rho_{x-1}^{n-j}\|\mathbf{z}\|^{x}
$$

для всех $j=0,1, \ldots, n$. Таким образом,

$$
\begin{aligned}
\mathbf{E}\|\mathbf{Z}(n)\|^{x} \leqslant & (1+\varepsilon)^{n} L_{x}(s(x)(1+\delta))^{n}\|\mathbf{z}\|^{x} \\
& +c_{x, \varepsilon} R_{x} m^{x} M_{x} L_{x} K_{x-1}\|\mathbf{z}\|^{x} \sum_{j=0}^{n-1}(1+\varepsilon)^{j}(s(x)(1+\delta))^{j} \rho_{x-1}^{n-j-1} \\
= & L_{x} s^{n}(x)(1+\varepsilon)^{n}(1+\delta)^{n}\|\mathbf{z}\|^{x} \\
& +K s^{n}(x)(1+\varepsilon)^{n}(1+\delta)^{n}\|\mathbf{z}\|^{x} \sum_{j=1}^{n} \frac{\rho_{x-1}^{j-1}}{s^{j}(x)(1+\varepsilon)^{j}(1+\delta)^{j}} .
\end{aligned}
$$

Выбирая теперь $\delta$ и $\varepsilon$ так, чтобы

$$
s(x)(1+\varepsilon)(1+\delta) \in\left(\rho_{x-1}, 1\right)
$$

(последнее всегда возможно, поскольку $\rho_{x-1}<s(x)<1$ ), приходим к (23). 
Для анализа нецелых значений $x \in[1, \kappa)$ заметим, что $x^{*}=[\kappa] \in$ $[1, \kappa)$ является целым числом, для которого оценка (23) справедлива. Таким образом, осталось проверить (23) для $x=x^{*}+\gamma<\kappa$, где $\gamma \in(0,1)$. Поскольку $x^{*} \geqslant x / 2 \vee 1$, то можно использовать такие же рассуждения, как и ранее, заменив в них $x-1$ на $x^{*}$.

Лемма доказана.

Для натурального числа $r$ положим

$$
\zeta=\zeta(r):=\min \{n \geqslant 0:\|\mathbf{Z}(n)\|>r\}
$$

с естественным соглашением $\zeta=\infty$, если $\max _{n}\|\mathbf{Z}(n)\| \leqslant r$.

Лемма 3. Если выполнень условия теоремь 2, то для любого фиксированного $r \geqslant 1$ справедлива оченка

$$
\mathbf{E}\left[\|\mathbf{Z}(\zeta(r))\|^{\kappa} I\{\zeta(r)<\infty\}\right]<\infty .
$$

Д о к а з а т е л ь с т в о. Аналогично (28) для $x>1$ имеем

$$
\begin{array}{r}
\mathbf{E}_{\mathbf{F}}\left[\|\mathbf{Z}(n)\|^{x} \mid \mathbf{Z}(0), \ldots, \mathbf{Z}(n-1)\right] \leqslant(1+\varepsilon)\left\|\mathbf{Z}(n-1) A_{n-1}\right\|^{x} \\
+c_{x, \varepsilon} R_{x} m^{x} \sum_{i=1}^{m} M_{x}(n-1, i) Z_{i}^{x / 2 \vee 1}(n-1)=: \Psi_{x}(n-1),
\end{array}
$$

в то время как неравенство Иенсена приводит при $x \leqslant 1$ к оценке

$$
\begin{aligned}
\mathbf{E}_{\mathbf{F}}\left[\|\mathbf{Z}(n)\|^{x} \mid \mathbf{Z}(0), \ldots, \mathbf{Z}(n-1)\right] & \leqslant\left(\mathbf{E}_{\mathbf{F}}[\|\mathbf{Z}(n)\| \mid \mathbf{Z}(0), \ldots, \mathbf{Z}(n-1)]\right)^{x} \\
& =\left\|\mathbf{Z}(n-1) A_{n-1}\right\|^{x} \leqslant \Psi_{x}(n-1) .
\end{aligned}
$$

Ясно, что

$$
\begin{aligned}
\Psi_{\kappa}(n-1) I\{n<\zeta\} & \leqslant Q_{n-1}(r) \\
& :=(1+\varepsilon) r^{\kappa}\left\|A_{n-1}\right\|^{\kappa}+c_{\kappa, \varepsilon} R_{\kappa} m^{\kappa} r^{\kappa / 2 \vee 1} \sum_{i=1}^{m} M_{\kappa}(\zeta-1, i) .
\end{aligned}
$$

Используя эти соотношения, нетрудно понять, что на событии $\{\zeta<\infty\}$ справедливы оценки

$$
\begin{aligned}
\|\mathbf{Z}(\zeta)\|^{\kappa} & =\Psi_{\kappa}(\zeta-1) \frac{\|\mathbf{Z}(\zeta)\|^{\kappa}}{\Psi_{\kappa}(\zeta-1)} \leqslant Q_{\zeta-1}(r) \frac{\|\mathbf{Z}(\zeta)\|^{\kappa}}{\Psi_{\kappa}(\zeta-1)} \\
& \leqslant \sum_{\zeta \leqslant n<\tau} Q_{n}(r) \frac{\|\mathbf{Z}(n)\|^{\kappa}}{\Psi_{\kappa}(n-1)} .
\end{aligned}
$$

В силу (31) и (32)

$$
\mathbf{E}\left[\|Z(\zeta)\|^{\kappa} I\{\zeta<\infty\}\right] \leqslant \sum_{n \geqslant 1} \mathbf{E}\left[Q_{n}(r) \frac{\|\mathbf{Z}(n)\|^{\kappa}}{\Psi_{\kappa}(n-1)} I\{\tau \geqslant n\}\right]
$$




$$
\begin{aligned}
& \leqslant \sum_{n \geqslant 1} \mathbf{E}\left[Q_{n}(r) I\{\tau \geqslant n\}\right]=\sum_{n \geqslant 1} \mathbf{P}(\tau \geqslant n) \mathbf{E Q}_{\mathbf{n}}(\mathbf{r}) \\
& =\left[(1+\varepsilon) r^{\kappa} \mathbf{E}\|A\|^{\kappa}+c_{\kappa, \varepsilon} R_{\kappa} m^{\kappa+1} r^{\kappa / 2 \vee 1} M_{\kappa}\right] \sum_{n \geqslant 1} \mathbf{P}(\tau \geqslant n)<\infty,
\end{aligned}
$$

поскольку при $\kappa_{1}=\min (\kappa / 2,1)$

$$
\begin{aligned}
\sum_{n \geqslant 1} \mathbb{P}(\tau \geqslant n) & =\sum_{n \geqslant 1} \mathbf{P}(\|\mathbf{Z}(n-1)\| \geqslant 1)=\sum_{n \geqslant 1} \mathbf{E}\left[\mathbf{P}_{\mathbf{F}}(\|\mathbf{Z}(n-1)\| \geqslant 1)\right] \\
& \leqslant \sum_{n \geqslant 1} \mathbf{E}\left[\mathbf{E}_{\mathbf{F}}\|\mathbf{Z}(n-1)\|^{\kappa_{1}}\right] \leqslant \sum_{n \geqslant 1} \mathbf{E}\left\|\mathbf{E}_{\mathbf{F}} \mathbf{Z}(n-1)\right\|^{\kappa_{1}} \\
& \leqslant \sum_{n \geqslant 1}\|z\|^{\kappa_{1}} \mathbf{E}\left\|A_{0} \cdots A_{n-1}\right\|^{\kappa_{1}}<\infty .
\end{aligned}
$$

Лемма доказана.

Пусть $\mathscr{B}_{n}(n=1,2, \ldots)$ обозначает $\sigma$-алгебру, порожденную набором

$$
\mathbf{F}_{0}(\cdot, \cdot), \mathbf{F}_{1}(\cdot, \cdot), \ldots, \mathbf{F}_{n-1}(\cdot, \cdot), \mathbf{Z}(0), \ldots, \mathbf{Z}(n),
$$

и пусть

$$
\mathbf{C}_{n}:=\left(\mathbf{E}_{\mathbf{F}} \varphi_{1}(n), \ldots, \mathbf{E}_{\mathbf{F}} \varphi_{m}(n)\right)^{\prime}, \quad n=0,1,2, \ldots
$$

Напомним, что, согласно определению интересующего нас ВПСС, пары $\left(A_{n}, \mathbf{C}_{n}\right), n=0,1, \ldots$, независимы и одинаково распределены. В частности, пара $\left(A_{n}, \mathbf{C}_{n}\right)$ не зависит от $\mathscr{B}_{n}$. Положим

$$
S(\zeta):=\sum_{n=\zeta}^{\infty}\left\langle\mathbf{Z}(n), \mathbf{C}_{n}\right\rangle
$$

Следующая лемма показывает, что для больших значений параметра $r$ случайная величина $S(\zeta)=S(\zeta(r))$ близка, в некотором смысле, к условному математическому ожиданию $\mathbf{E}\left[S(\zeta) \mid \mathscr{B}_{\zeta}\right]=\left\langle\mathbf{Z}(\zeta), \Xi_{\zeta}\right\rangle$.

Лемма 4. Если выполнены условия теоремы 2, то для любого $\varepsilon>0$ найдется $r=r(\varepsilon)$ maкое, что для $\zeta=\zeta(r)$ и всех $y \geqslant y_{0}$

$$
\mathbf{P}\left(\left|S(\zeta)-\left\langle\mathbf{Z}(\zeta), \Xi_{\zeta}\right\rangle\right|>\varepsilon y ; \zeta<\infty\right) \leqslant \frac{\varepsilon}{y^{\kappa}} \mathbf{E}\left[\|\mathbf{Z}(\zeta)\|^{\kappa} I\{\zeta<\infty\}\right] .
$$

Д о к а з а т е л ь с т в о. Очевидно, что при $n \geqslant \zeta+1$

$$
\begin{aligned}
\left\langle\mathbf{Z}(n)-\mathbf{Z}(\zeta) \Pi_{\zeta, n}, \mathbf{C}_{n}\right\rangle & =\sum_{l=\zeta+1}^{n}\left\langle\mathbf{Z}(l) \Pi_{l, n}-\mathbf{Z}(l-1) \Pi_{l-1, n}, \mathbf{C}_{n}\right\rangle \\
& =\sum_{l=\zeta+1}^{n}\left\langle\mathbf{Z}(l)-\mathbf{Z}(l-1) A_{l-1}, \Pi_{l, n} \mathbf{C}_{n}\right\rangle
\end{aligned}
$$


что влечет соотношения

$$
\begin{aligned}
\left|S(\zeta)-\left\langle\mathbf{Z}(\zeta), \Xi_{\zeta}\right\rangle\right| & =\left|\sum_{n=\zeta+1}^{\infty}\left\langle\mathbf{Z}(n)-\mathbf{Z}(\zeta) \Pi_{\zeta, n}, \mathbf{C}_{n}\right\rangle\right| \\
& \leqslant \sum_{n=\zeta+1}^{\infty}\left|\left\langle\mathbf{Z}(n)-\mathbf{Z}(\zeta) \Pi_{\zeta, n}, \mathbf{C}_{n}\right\rangle\right| \\
& =\sum_{n=\zeta+1}^{\infty}\left|\sum_{l=\zeta+1}^{n}\left\langle\mathbf{Z}(l)-\mathbf{Z}(l-1) A_{l-1}, \Pi_{l, n} \mathbf{C}_{n}\right\rangle\right| \\
& \leqslant \sum_{l=\zeta+1}^{\infty} \sum_{n=l}^{\infty}\left|\left\langle\mathbf{Z}(l)-\mathbf{Z}(l-1) A_{l-1}, \Pi_{l, n} \mathbf{C}_{n}\right\rangle\right| \\
& \leqslant \sum_{l=\zeta+1}^{\infty}\left\|\mathbf{Z}(l)-\mathbf{Z}(l-1) A_{l-1}\right\|\left\|\sum_{n=l}^{\infty} \Pi_{l, n} \mathbf{C}_{n}\right\| \\
& =\sum_{l=\zeta+1}^{\infty}\left\|\mathbf{Z}(l)-\mathbf{Z}(l-1) A_{l-1}\right\|\left\|\Xi_{l}\right\| .
\end{aligned}
$$

Отсюда, отталкиваясь от равенства $\sum_{j=1}^{\infty} j^{-2}=\pi^{2} / 6 \leqslant 2$ и применяя на событии $\{\zeta<\infty\}$ рассуждения, использовавшиеся в лемме 3 работы [29], заключаем, что

$$
\begin{aligned}
& \mathbf{P}\left(\left|S(\zeta)-\left\langle\mathbf{Z}(\zeta), \Xi_{\zeta}\right\rangle\right| \geqslant \varepsilon y \mid \mathscr{B}_{\zeta}\right) \\
& \quad \leqslant \mathbf{P}\left(\sum_{l=\zeta+1}^{\infty}\left\|\mathbf{Z}(l)-\mathbf{Z}(l-1) A_{l-1}\right\|\left\|\Xi_{l}\right\| \geqslant 6 \pi^{-2} \varepsilon y \sum_{l=\zeta+1}^{\infty} \frac{1}{(l-\zeta)^{2}} \mid \mathscr{B}_{\zeta}\right) \\
& \quad \leqslant \sum_{l=\zeta+1}^{\infty} \mathbf{P}\left(\left\|\mathbf{Z}(l)-\mathbf{Z}(l-1) A_{l-1}\right\|\left\|\Xi_{l}\right\| \geqslant \frac{\varepsilon y}{2(l-\zeta)^{2}} \mid \mathscr{B}_{\zeta}\right) .
\end{aligned}
$$

Поскольку случайные векторы $\mathbf{Z}(l)-\mathbf{Z}(l-1) A_{l-1}$ и $\Xi_{l}$ независимы при осуществлении события $\{\zeta \leqslant l<\infty\}$, то

$$
\begin{aligned}
& \mathbf{P}\left(\left\|\mathbf{Z}(l)-\mathbf{Z}(l-1) A_{l-1}\right\|\left\|\Xi_{l}\right\| \geqslant \frac{\varepsilon y}{2(l-\zeta)^{2}} \mid \mathscr{B}_{\zeta}\right) \\
& \quad=\int_{0}^{\infty} \mathbf{P}\left(\left\|\mathbf{Z}(l)-\mathbf{Z}(l-1) A_{l-1}\right\| \in d t \mid \mathscr{B}_{\zeta}\right) \mathbf{P}\left(\|\Xi\| \geqslant \frac{\varepsilon y}{2 t(l-\zeta)^{2}} \mid \mathscr{B}_{\zeta}\right) .
\end{aligned}
$$

Согласно условию Т, существует константа $K \in(0, \infty)$ такая, что при всех $l>\zeta$

$$
\begin{aligned}
& \mathbf{P}\left(\left\|\mathbf{Z}(l)-\mathbf{Z}(l-1) A_{l-1}\right\|\left\|\Xi_{l}\right\| \geqslant \frac{\varepsilon y}{2(l-\zeta)^{2}} \mid \mathscr{B}_{\zeta}\right) \\
& \quad \leqslant \int_{0}^{\infty} \mathbf{P}\left(\left\|\mathbf{Z}(l)-\mathbf{Z}(l-1) A_{l-1}\right\| \in d t \mid \mathscr{B}_{\zeta}\right) \frac{K t^{\kappa}}{\varepsilon^{\kappa} y^{\kappa}}(l-\zeta)^{2 \kappa} \\
& \quad \leqslant \frac{K}{\varepsilon^{\kappa} y^{\kappa}}(l-\zeta)^{2 \kappa} \mathbf{E}\left[\left\|\mathbf{Z}(l)-\mathbf{Z}(l-1) A_{l-1}\right\|^{\kappa} \mid \mathscr{B}_{\zeta}\right] .
\end{aligned}
$$


Рассмотрим теперь отдельно случаи $\kappa \leqslant 1$ и $\kappa>1$.

В первом случае воспользуемся неравенством

$$
\mathbf{E}\left[\left\|\mathbf{Z}(l)-\mathbf{Z}(l-1) A_{l-1}\right\|^{\kappa} \mid \mathscr{B}_{\zeta}\right] \leqslant\left(\mathbf{E}\left[\left\|\mathbf{Z}(l)-\mathbf{Z}(l-1) A_{l-1}\right\|^{2} \mid \mathscr{B}_{\zeta}\right]\right)^{\kappa / 2} .
$$

Далее, имеем

$$
\begin{aligned}
\mathbf{E} & {\left[\left\|\mathbf{Z}(l)-\mathbf{Z}(l-1) A_{l-1}\right\|^{2} \mid \mathscr{B}_{\zeta}\right] } \\
& =\mathbf{E}\left[\left(\sum_{i=1}^{m} \sum_{k=1}^{Z_{i}(l-1)} \sum_{j=1}^{m}\left[\boldsymbol{\xi}_{i j}(l-1 ; k)-a_{i j}(l-1)\right]\right)^{2} \mid \mathscr{B}_{\zeta}\right] \\
& =\sum_{i=1}^{m} \mathbf{E}_{\mathbf{F}} \beta_{i}^{2}(l-1) \mathbf{E}\left[Z_{i}(l-1) \mid \mathscr{B}_{\zeta}\right] \\
& =\sum_{i=1}^{m} \mathbf{E}_{\mathbf{F}} \beta_{i}^{2}(l-1)\left(\mathbf{Z}(\zeta) \Pi_{\zeta, l-1}\right)_{i} \leqslant\left\|\mathbf{Z}(\zeta) \Pi_{\zeta, l-1}\right\| \sum_{i=1}^{m} \mathbf{E}_{\mathbf{F}} \beta_{i}^{2}(l-1) .
\end{aligned}
$$

Таким образом, при $\kappa \leqslant 1$

$$
\left(\mathbf{E}\left[\left\|\mathbf{Z}(l)-\mathbf{Z}(l-1) A_{l-1}\right\|^{2} \mid \mathscr{B}_{\zeta}\right]\right)^{\kappa / 2} \leqslant\left\|\mathbf{Z}(\zeta) \Pi_{\zeta, l-1}\right\|^{\kappa / 2}\left(\sum_{i=1}^{m} \mathbf{E}_{\mathbf{F}} \beta_{i}^{2}(l-1)\right)^{\kappa / 2} .
$$

Это, в силу неравенства

$$
s\left(\frac{\kappa}{2}\right)=\lim _{n \rightarrow \infty}\left(\mathbf{E}\left\|\Pi_{0, n}\right\|^{\kappa / 2}\right)^{1 / n}<1,
$$

первой части условия (22) и соотношений (37)-(41), приводит к оценке

$$
\begin{aligned}
& \mathbf{P}\left(\left|S(\zeta)-\left\langle\mathbf{Z}(\zeta), \Xi_{\zeta}\right\rangle\right|>\varepsilon y ; \zeta<\infty\right) \\
& \quad \leqslant \frac{K}{\varepsilon^{\kappa} y^{\kappa}} \mathbf{E}\left[\sum_{l=\zeta+1}^{\infty}(l-\zeta)^{2 \kappa}\|\mathbf{Z}(\zeta)\|^{\kappa / 2}\left\|\Pi_{\zeta, l-1}\right\|^{\kappa / 2} I\{\zeta<\infty\}\right] \\
& \quad=\frac{K}{\varepsilon^{\kappa} y^{\kappa}} \mathbf{E}\left[\|\mathbf{Z}(\zeta)\|^{\kappa / 2} I\{\zeta<\infty\} \sum_{l=1}^{\infty} l^{2 \kappa} \mathbf{E}\left\|\Pi_{0, l-1}\right\|^{\kappa / 2}\right] \\
& \quad \leqslant \frac{\text { const }}{\varepsilon^{\kappa} y^{\kappa} r^{\kappa / 2}} \mathbf{E}\left[\|\mathbf{Z}(\zeta)\|^{\kappa} I\{\zeta<\infty\}\right] \leqslant \frac{\varepsilon}{y^{\kappa}} \mathbf{E}\left[\|\mathbf{Z}(\zeta)\|^{\kappa} I\{\zeta<\infty\}\right],
\end{aligned}
$$

справедливой для всех $r \geqslant r_{0}(\varepsilon)$, что доказывает лемму при $\kappa \leqslant 1$.

При $\kappa>1$ воспользуемся леммой 1 , что для любого $l>\zeta$ приводит к соотношениям

$$
\begin{aligned}
\mathbf{E} & {\left[\left\|\mathbf{Z}(l)-\mathbf{Z}(l-1) A_{l-1}\right\|^{\kappa} \mid \mathscr{B}_{\zeta}\right] } \\
& \leqslant R_{\kappa} m^{\kappa} \sum_{i=1}^{m} M_{\kappa}(n ; i) \mathbf{E}\left[\left\|Z_{i}(l-1)\right\|^{\kappa / 2 \vee 1} \mid \mathscr{B}_{\zeta}\right] \\
& \leqslant R_{\kappa} m^{\kappa} \mathbf{E}\left[\|\mathbf{Z}(l-1)\|^{\kappa / 2 \vee 1} \mid \mathscr{B}_{\zeta}\right] \sum_{i=1}^{m} M_{\kappa}(n ; i) .
\end{aligned}
$$


Согласно лемме 2 , найдутся константы $\rho_{\kappa / 2 \vee 1} \in(0,1)$ и $K_{\kappa / 2 \vee 1}<\infty$ такие, что при всех $l>\zeta$

$$
\mathbf{E}\left[\|\mathbf{Z}(l-1)\|^{\kappa / 2 \vee 1} \mid \mathscr{B}_{\zeta}\right] \leqslant K_{\kappa / 2 \vee 1} \rho_{\kappa / 2 \vee 1}^{l-\zeta-1}\|\mathbf{Z}(\zeta)\|^{\kappa / 2 \vee 1} .
$$

Следовательно,

$$
\begin{aligned}
& \mathbf{P}\left(\left|S(\zeta)-\left\langle\mathbf{Z}(\zeta), \Xi_{\zeta}\right\rangle\right|>\varepsilon y ; \zeta<\infty\right) \\
& \quad \leqslant \frac{m^{\kappa} K}{\varepsilon^{\kappa} y^{\kappa}} \mathbf{E}\left[\sum_{l=\zeta+1}^{\infty}(l-\zeta)^{2 \kappa} \mathbf{E}\left[\|\mathbf{Z}(l-1)\|^{\kappa / 2 \vee 1} \sum_{i=1}^{m} M_{\kappa}(n ; i) \mid \mathscr{B}_{\zeta}\right] I\{\zeta<\infty\}\right] \\
& \quad \leqslant \frac{m^{\kappa+1} K M_{\kappa}}{\varepsilon^{\kappa} y^{\kappa}} \mathbf{E}\left[\sum_{l=\zeta+1}^{\infty}(l-\zeta)^{2 \kappa} \mathbf{E}\left[\|\mathbf{Z}(l-1)\|^{\kappa / 2 \vee 1} \mid \mathscr{B}_{\zeta}\right] I\{\zeta<\infty\}\right] \\
& \quad \leqslant \frac{m^{\kappa+1} K M_{\kappa}}{\varepsilon^{\kappa} y^{\kappa}} \mathbf{E}\left[\sum_{l=\zeta+1}^{\infty}(l-\zeta)^{2 \kappa}\|\mathbf{Z}(\zeta)\|^{\kappa / 2 \vee 1} K_{\kappa / 2 \vee 1} \rho_{\kappa / 2 \vee 1}^{l-\zeta-1} I\{\zeta<\infty\}\right] \\
& \quad=\frac{m^{\kappa+1} K M_{\kappa} K_{\kappa / 2 \vee 1}}{\varepsilon^{\kappa} y^{\kappa}}\left[\|\mathbf{Z}(\zeta)\|^{\kappa / 2 \vee 1} I\{\zeta<\infty\} \sum_{l=1}^{\infty} l^{2 \kappa} \rho_{\kappa / 2 \vee 1}^{l-1}\right] \\
& \quad \leqslant \frac{\operatorname{const}}{\varepsilon^{\kappa} y^{\kappa} r^{\kappa-\kappa / 2 \vee 1}} \mathbf{E}\left[\|\mathbf{Z}(\zeta)\|^{\kappa} I\{\zeta<\infty\}\right] \leqslant \frac{\varepsilon}{y^{\kappa}} \mathbf{E}\left[\|\mathbf{Z}(\zeta)\|^{\kappa} I\{\zeta<\infty\}\right]
\end{aligned}
$$

(последнее справедливо, если $r$ выбрано достаточно большим), что оправдывает утверждение леммы при $\kappa>1$.

Лемма доказана.

6. Накопленный объем финального продукта. В этом разделе мы получим некоторые оценки, связанные с общим объемом финального продукта, накопленного в докритическом ВПСС за время его эволюции.

Обозначим

$$
\Delta(n):=\sum_{i=1}^{m} \sum_{k=1}^{Z_{i}(n)} \varphi_{i}(n ; k)
$$

общий объем финального продукта, произведенного частицами $n$-го поколения ВПСС и положим

$$
\bar{\Phi}(N):=\sum_{n=N}^{\infty} \Delta(n), \quad \Phi=\bar{\Phi}(0) .
$$

Лемма 5. Если выполнень условия теоремь 2, то для любого $\varepsilon>0$ найдется число $r=r(\varepsilon)$ такое, ито

$$
\mathbf{P}(|\bar{\Phi}(\zeta)-S(\zeta)|>\varepsilon y ; \zeta<\infty) \leqslant \frac{\varepsilon}{y^{\kappa}} \mathbf{E}\left[\|\mathbf{Z}(\zeta)\|^{\kappa} I\{\zeta<\infty\}\right]
$$

$\partial \Omega я$ всех $y \geqslant y_{0}$. 
Д о к а з а т е ль с т в о. Имеем

$$
\Delta(n)-\left\langle\mathbf{Z}(n), \mathbf{C}_{n}\right\rangle=\sum_{i=1}^{m} \sum_{k=1}^{Z_{i}(n)}\left(\varphi_{i}(n ; k)-\mathbf{E}_{\mathbf{F}} \varphi_{i}(n)\right) .
$$

Рассмотрим отдельно случаи $\kappa \leqslant 1$ и $\kappa>1$.

Используя при $\kappa \geqslant 1$ леммы 1 и 2 , получаем для $n>\zeta$ :

$$
\begin{aligned}
& \mathbf{P}\left(\left|\Delta(n)-\left\langle\mathbf{Z}(n), \mathbf{C}_{n}\right\rangle\right|>\frac{\varepsilon y}{2(n-\zeta)^{2}} \mid \mathscr{B}_{\zeta}\right) \\
& \quad \leqslant \frac{4^{\kappa}(n-\zeta)^{2 \kappa}}{(\varepsilon y)^{\kappa}} \mathbf{E}\left[\left|\sum_{i=1}^{m} \sum_{k=1}^{Z_{i}(n)}\left(\varphi_{i}(n ; k)-\mathbf{E}_{\mathbf{F}} \varphi_{i}(n)\right)\right|^{\kappa} \mid \mathscr{B}_{\zeta}\right] \\
& \quad \leqslant(4 m)^{\kappa} R_{\kappa} \frac{(n-\zeta)^{2 \kappa}}{(\varepsilon y)^{\kappa}} \sum_{i=1}^{m} \mathbf{E}_{\mathbf{F}}\left|\varphi_{i}(n)-\mathbf{E}_{\mathbf{F}} \varphi_{i}(n)\right|^{\kappa} \mathbf{E}\left[\|\mathbf{Z}(n)\|^{\kappa / 2 \vee 1} \mid \mathscr{B}_{\zeta}\right] \\
& \quad \leqslant(4 m)^{\kappa} R_{\kappa} K_{\kappa / 2 \vee 1} \frac{(n-\zeta)^{2 \kappa}}{(\varepsilon y)^{\kappa}} \sum_{i=1}^{m} \mathbf{E}_{\mathbf{F}}\left|\varphi_{i}(n)-\mathbf{E}_{\mathbf{F}} \varphi_{i}(n)\right|^{\kappa}\|\mathbf{Z}(\zeta)\|^{\kappa / 2 \vee 1} \rho_{\kappa / 2 \vee 1}^{n-\zeta} .
\end{aligned}
$$

В силу условия (21) отсюда следуют оценки

$$
\begin{aligned}
& \mathbf{P}(|\bar{\Phi}(\zeta)-S(\zeta)|>\varepsilon y ; \zeta<\infty) \\
& \quad \leqslant \mathbf{E}\left[\sum_{n=\zeta}^{\infty} \mathbf{P}\left(\left|\Delta(n)-\left\langle\mathbf{Z}(n), \mathbf{C}_{n}\right\rangle\right|>\frac{\varepsilon y}{2(n-\zeta)^{2}} \mid \mathscr{B}_{\zeta}\right) I\{\zeta<\infty\}\right] \\
& \quad \leqslant \frac{(4 m)^{\kappa} R_{\kappa} K_{\kappa / 2 \vee 1} K}{(\varepsilon y)^{\kappa}} \mathbf{E}\left[\|\mathbf{Z}(\zeta)\|^{\kappa / 2 \vee 1} \sum_{n=\zeta}^{\infty}(n-\zeta)^{2 \kappa} \rho_{\kappa / 2 \vee 1}^{n-\zeta} I\{\zeta<\infty\}\right] \\
& \quad=\frac{\text { const }}{(\varepsilon y)^{\kappa}} \mathbf{E}\left[\|\mathbf{Z}(\zeta)\|^{\kappa / 2 \vee 1} I\{\zeta<\infty\}\right] \sum_{n=1}^{\infty} n^{2 \kappa} \rho_{\kappa / 2}^{n}{ }^{n} 1 \\
& \quad \leqslant \frac{\operatorname{const}}{(\varepsilon y)^{\kappa} r^{\kappa-(\kappa / 2 \vee 1)}} \mathbf{E}\left[\|\mathbf{Z}(\zeta)\|^{\kappa} I\{\zeta<\infty\}\right] \leqslant \frac{\varepsilon}{y^{\kappa}} \mathbf{E}\left[\|\mathbf{Z}(\zeta)\|^{\kappa} I\{\zeta<\infty\}\right],
\end{aligned}
$$

справедливые при $r \geqslant r(\varepsilon)$.

При $\kappa \leqslant 1$ и $n>\zeta$ имеет место неравенство

$$
\mathbf{E}\left[\left|\Delta(n)-\left\langle\mathbf{Z}(n), \mathbf{C}_{n}\right\rangle\right|^{\kappa} \mid \mathscr{B}_{\zeta}\right] \leqslant\left(\mathbf{E}\left[\left|\Delta(n)-\left\langle\mathbf{Z}(n), \mathbf{C}_{n}\right\rangle\right|^{2} \mid \mathscr{B}_{\zeta}\right]\right)^{\kappa / 2} .
$$

Очевидно, что

$$
\begin{aligned}
& \mathbf{E}\left[\left|\Delta(n)-\left\langle\mathbf{Z}(n), \mathbf{C}_{n}\right\rangle\right|^{2} \mid \mathscr{B}_{\zeta}\right] \leqslant m^{2} \sum_{i=1}^{m} \mathbf{E}_{\mathbf{F}} \beta_{i}^{2}(n) \mathbf{E}\left[Z_{i}(n) \mid \mathscr{B}_{\zeta}\right] \\
& =m^{2} \sum_{i=1}^{m} \mathbf{E}_{\mathbf{F}} \beta_{i}^{2}(n)\left(\mathbf{Z}(\zeta) \Pi_{\zeta, n}\right)_{i} \leqslant m^{2}\left\|\mathbf{Z}(\zeta) \Pi_{\zeta, n}\right\| \sum_{i=1}^{m} \mathbf{E}_{\mathbf{F}} \beta_{i}^{2}(n) .
\end{aligned}
$$


Таким образом, при $\kappa \leqslant 1$

$$
\left(\mathbf{E}\left[\left|\Delta(n)-\left\langle\mathbf{Z}(n), \mathbf{C}_{n}\right\rangle\right|^{2} \mid \mathscr{B}_{\zeta}\right]\right)^{\kappa / 2} \leqslant m^{\kappa}\left\|\mathbf{Z}(\zeta) \Pi_{\zeta, n+1}\right\|^{\kappa / 2}\left(\sum_{i=1}^{m} \mathbf{E}_{\mathbf{F}} \beta_{i}^{2}(n)\right)^{\kappa / 2} .
$$

Эта оценка в сочетании с условием (22) показывает, что

$$
\begin{aligned}
& \mathbf{P}(|\bar{\Phi}(\zeta)-S(\zeta)|>\varepsilon y ; \zeta<\infty) \\
& \leqslant \mathbf{E}\left[\sum_{n=\zeta}^{\infty} \mathbf{P}\left(\left|\Delta(n)-\left\langle\mathbf{Z}(n), \mathbf{C}_{n}\right\rangle\right|>\frac{\varepsilon y}{2(n-\zeta)^{2}} \mid \zeta, \mathbf{Z}(0), \ldots, \mathbf{Z}(\zeta)\right) I\{\zeta<\infty\}\right] \\
& \leqslant \frac{m^{\kappa} K}{\varepsilon^{\kappa} y^{\kappa}} \mathbf{E}\left[\sum_{l=\zeta+1}^{\infty}(l-\zeta)^{2 \kappa}\|\mathbf{Z}(\zeta)\|^{\kappa / 2}\left\|\Pi_{\zeta, l}\right\|^{\kappa / 2} I\{\zeta<\infty\}\right] \\
& =\frac{m^{\kappa} K}{\varepsilon^{\kappa} y^{\kappa}} \mathbf{E}\left[\|\mathbf{Z}(\zeta)\|^{\kappa / 2} \sum_{l=1}^{\infty} l^{2 \kappa} \mathbf{E}\left\|\Pi_{0, l}\right\|^{\kappa / 2} I\{\zeta<\infty\}\right] \\
& \leqslant \frac{\operatorname{const}}{\varepsilon^{\kappa} y^{\kappa} r^{\kappa / 2}} \mathbf{E}\left[\|\mathbf{Z}(\zeta)\|^{\kappa} I\{\zeta<\infty\}\right] \leqslant \frac{\varepsilon}{y^{\kappa}} \mathbf{E}\left[\|\mathbf{Z}(\zeta)\|^{\kappa} I\{\zeta<\infty\}\right]
\end{aligned}
$$

для всех $r \geqslant r(\varepsilon)$.

Лемма доказана.

До настоящего момента мы предполагали, что начальное число частиц в процессе и объем финального продукта неслучайны. В леммах 6 и 7 мы отказываемся от этого допущения.

Лемма 6. Если ВПСС докритический, $\mathbf{E}\|\mathbf{Z}(0)\|<\infty$ и найдется число $\delta>0$ такое, что

$$
\mathbf{E} \Phi^{\kappa+\delta}(0)<\infty, \quad \max _{1 \leqslant i \leqslant m} \mathbf{E} \varphi_{i}^{\kappa+\delta}<\infty,
$$

то для любого $\varepsilon>0$ существует $r=r(\varepsilon)$ такое, ито для $\xi=\xi(r)$

$$
\mathbf{P}(\Phi(\zeta-1)>\varepsilon y ; \quad \zeta<\infty)<\frac{1}{y^{\kappa+\delta / 2}}
$$

при всех $y \geqslant y_{0}$.

Д о к а з а т е л ь с т в о. Пусть, как и прежде, $\tau$ обозначает момент вырождения ВПСС, и пусть константа $c$ такова, что

$$
\sum_{t>c \ln y} \mathbf{P}(\tau>t) \leqslant \frac{1}{y^{\kappa+\delta}}
$$

для всех $y \geqslant y_{0}(c)$. В силу (15) такая константа, очевидно, найдется. Вспоминая (7), положим

$$
\Phi_{r}(n)=\Phi(0)+\sum_{l=0}^{n} \sum_{i=1}^{m} \sum_{k=1}^{r} \varphi_{i}(l ; k) .
$$


Для $y \geqslant y_{0}(c)$ имеем

$$
\begin{aligned}
\mathbf{P}( & (\zeta-1)>\varepsilon y ; \zeta<\infty)=\sum_{n=1}^{\infty} \mathbf{P}(\Phi(n-1)>\varepsilon y ; \zeta=n) \\
\leqslant & \sum_{n=1}^{\infty} \mathbf{P}\left(\Phi_{r}(n-1)>\varepsilon y ; \zeta=n\right) \leqslant \sum_{n=1}^{\infty} \mathbf{P}\left(\Phi_{r}(n-1)>\varepsilon y ; \tau>n\right) \\
\leqslant & \mathbf{P}\left(\Phi(0)>\frac{\varepsilon y}{2}\right) \mathbf{E} \tau+\sum_{1 \leqslant n \leqslant c \ln } \sum_{l=0}^{n} \sum_{i=1}^{m} \sum_{k=1}^{r} \mathbf{P}\left(\varphi_{i}(l ; k)>\frac{\varepsilon y}{2 m r c^{2} \ln ^{2} y}\right) \\
& +\sum_{n>c \ln y} \mathbf{P}(\tau>n) \\
\leqslant & \left(\frac{2}{\varepsilon y}\right)^{\kappa+\delta} \mathbf{E} \Phi^{\kappa+\delta}(0) \mathbf{E} \tau \\
& +\frac{\left(2 m r c^{2} \ln ^{2} y\right)^{\kappa+\delta}}{(\varepsilon y)^{\kappa+\delta}} \sum_{1 \leqslant n \leqslant c l n} \sum_{l=0}^{n} \sum_{i=1}^{m} \sum_{k=1}^{r} \mathbf{E} \varphi_{i}^{\kappa+\delta}+\frac{1}{y^{\kappa+\delta}} \\
\leqslant & K \frac{\ln ^{2(\kappa+\delta+1)} y}{(\varepsilon y)^{\kappa+\delta}}+\frac{1}{y^{\kappa+\delta}} \leqslant \frac{1}{y^{\kappa+\delta / 2}} .
\end{aligned}
$$

Лемма 7. В условиях леммы 6 для любого $r$ найдется число $y_{0}=$ $y_{0}(r)$ maкoe, umo

$$
\mathbf{P}(\Phi>y ; \quad \zeta=\infty) \leqslant \frac{1}{y^{\kappa+\delta / 2}}
$$

при всех $y>y_{0}$.

Д о к а з а т е ль с т в о. Для той же самой константы $c$, что и в предыдущей лемме, и $y \geqslant y_{0}(c)$ имеем

$$
\begin{aligned}
& \mathbf{P}(\Phi>y ; \zeta=\infty) \leqslant \mathbf{P}\left(\Phi_{r}(\tau)>y\right) \leqslant \mathbf{P}\left(\Phi_{r}([c \ln y])>y\right)+\mathbf{P}(\tau>c \ln y) \\
& \quad \leqslant \mathbf{P}\left(\Phi(0)>\frac{y}{2}\right)+\sum_{l=0}^{[c \ln y]} \sum_{i=1}^{m} \sum_{k=1}^{r} \mathbf{P}\left(\varphi_{i}(l ; k)>\frac{y}{2 m r[c \ln y]}\right)+\mathbf{P}(\tau>c \ln y) \\
& \quad \leqslant K \frac{\ln ^{\kappa+\delta+1} y}{y^{\kappa+\delta}}+\frac{1}{y^{\kappa+\delta}},
\end{aligned}
$$

что и требовалось.

7. Доказательство теоремы 2. Теперь мы можем приступить к доказательству основного результата работы, теоремы 2 . Заметим сначала, что в силу эквивалентности норм $\|\cdot\|$ и $\|\cdot\|_{2}$ и оценок (35), (43), (44) и (45) для любого $\varepsilon \in(0,1 / 3)$ найдется число $r=r(\varepsilon)$ такое, что при всех $y \geqslant y_{0}(r, \varepsilon)$ справедливы соотношения

$$
\begin{aligned}
& \mathbf{P}(\Phi>y) \leqslant \mathbf{P}(\Phi>y ; \zeta=\infty) \\
& \quad+\mathbf{P}\left(\left\langle\mathbf{Z}(\zeta), \Xi_{\zeta}\right\rangle>y(1-3 \varepsilon) ; \zeta<\infty\right)+\mathbf{P}(|\bar{\Phi}(\zeta)-S(\zeta)|>\varepsilon y ; \zeta<\infty)
\end{aligned}
$$




$$
\begin{aligned}
& +\mathbf{P}\left(\left|S(\zeta)-\left\langle\mathbf{Z}(\zeta), \Xi_{\zeta}\right\rangle\right|>\varepsilon y ; \zeta<\infty\right)+\mathbf{P}(\Phi(\zeta-1)>\varepsilon y ; \zeta<\infty) \\
\leqslant & \mathbf{P}\left(\left\langle\mathbf{Z}(\zeta), \Xi_{\zeta}\right\rangle>y(1-3 \varepsilon) ; \zeta<\infty\right) \\
& +\frac{2 \varepsilon}{y^{\kappa}} \mathbf{E}\left[\|\mathbf{Z}(\zeta)\|_{2}^{\kappa} I\{\zeta<\infty\}\right]+\frac{2}{y^{\kappa+\delta / 2}} .
\end{aligned}
$$

Пусть $U_{+}(r)=\left\{\mathbf{u}: r \leqslant\|\mathbf{u}\|_{2}<\infty\right\}$ и $K(l):=\inf _{\mathbf{u} \in U_{+}} l(\mathbf{u})>0$, где функция $l(\mathbf{u})$ та же, что и в условии Т. В силу этого условия и независимости случайных векторов $\mathbf{Z}(\zeta)$ и $\Xi_{\zeta}$, имеем

$$
\begin{aligned}
& \limsup _{y \rightarrow \infty} y^{\kappa} \mathbf{P}\left(\left\langle\mathbf{Z}(\zeta), \Xi_{\zeta}\right\rangle>y(1-3 \varepsilon) ; \zeta<\infty\right) \\
& \quad \leqslant \limsup _{y \rightarrow \infty} y^{\kappa} \int_{U_{+}(r)} \mathbf{P}(\mathbf{Z}(\zeta) \in d \mathbf{u} ; \zeta<\infty) \mathbf{P}\left(\left\langle\frac{\mathbf{u}}{\|\mathbf{u}\|_{2}}, \Xi\right\rangle \geqslant \frac{(1-3 \varepsilon) y}{\|\mathbf{u}\|_{2}}\right) \\
& \quad=K_{0}(1-3 \varepsilon)^{-\kappa} \int_{U_{+}(r)} \mathbf{P}(\mathbf{Z}(\zeta) \in d \mathbf{u} ; \zeta<\infty)\|\mathbf{u}\|_{2}^{\kappa} l\left(\frac{\mathbf{u}}{\|\mathbf{u}\|_{2}}\right) \\
& \quad=K_{0}(1-3 \varepsilon)^{-\kappa} \mathbf{E}\left[\|\mathbf{Z}(\zeta)\|_{2}^{\kappa} l\left(\frac{\mathbf{Z}(\zeta)}{\|\mathbf{Z}(\zeta)\|_{2}}\right) I\{\zeta<\infty\}\right]<\infty .
\end{aligned}
$$

Поскольку величина $y^{\kappa} \mathbf{P}(\Phi>y)$ не зависит от $r$ и $\varepsilon$, то предыдущая оценка и неравенства (46) влекут

$$
\limsup _{y \rightarrow \infty} y^{\kappa} \mathbf{P}(\Phi>y)<\infty
$$

и, более того,

$$
\limsup _{y \rightarrow \infty} y^{\kappa} \mathbf{P}(\Phi>y) \leqslant K_{0} \liminf _{r \rightarrow \infty} \mathbf{E}\left[\|\mathbf{Z}(\zeta)\|_{2}^{\kappa} l\left(\frac{\mathbf{Z}(\zeta)}{\|\mathbf{Z}(\zeta)\|_{2}}\right) I\{\zeta<\infty\}\right]
$$

Для получения аналогичной оценки снизу воспользуемся при $\varepsilon>0$ неравенством

$$
\begin{aligned}
\mathbf{P}(\Phi>y) \geqslant & \mathbf{P}(\bar{\Phi}(\zeta)>y ; \zeta<\infty) \geqslant \mathbf{P}\left(\left\langle\mathbf{Z}(\zeta), \Xi_{\zeta}\right\rangle>y(1+2 \varepsilon) ; \zeta<\infty\right) \\
& -\mathbf{P}(|\bar{\Phi}(\zeta)-S(\zeta)|>\varepsilon y ; \zeta<\infty) \\
& -\mathbf{P}\left(\left|S(\zeta)-\left\langle\mathbf{Z}(\zeta), \Xi_{\zeta}\right\rangle\right|>\varepsilon y ; \zeta<\infty\right) .
\end{aligned}
$$

Выберем теперь параметр $r$ столь большим, чтобы были справедливы оценки (35), (43) и (44). При таком выборе для достаточно больших значений $y>r$ имеет место неравенство

$$
\mathbf{P}(\Phi>y) \geqslant \mathbf{P}\left(\left\langle\mathbf{Z}(\zeta), \Xi_{\zeta}\right\rangle>y(1+2 \varepsilon) ; \zeta<\infty\right)-\frac{2 \varepsilon}{y^{\kappa}} \mathbf{E}\left[\|\mathbf{Z}(\zeta)\|_{2}^{\kappa} I\{\zeta<\infty\}\right] .
$$


Устремляя $y$ к бесконечности, получаем

$$
\begin{aligned}
& \liminf _{y \rightarrow \infty} y^{\kappa} \mathbf{P}\left(\left\langle\mathbf{Z}(\zeta), \Xi_{\zeta}\right\rangle>y(1+2 \varepsilon) ; \zeta<\infty\right) \\
& \quad=\liminf _{y \rightarrow \infty} y^{\kappa} \int_{U_{+}(r)} \mathbf{P}(\mathbf{Z}(\zeta) \in d \mathbf{u} ; \zeta<\infty) \mathbf{P}\left(\left\langle\frac{\mathbf{u}}{\|\mathbf{u}\|_{2}}, \Xi\right\rangle \geqslant \frac{(1+2 \varepsilon) y}{\|\mathbf{u}\|_{2}}\right) \\
& \quad=K_{0}(1+2 \varepsilon)^{-\kappa} \mathbf{E}\left[\|\mathbf{Z}(\zeta)\|_{2}^{\kappa} l\left(\frac{\mathbf{Z}(\zeta)}{\|\mathbf{Z}(\zeta)\|_{2}}\right) I\{\zeta<\infty\}\right] .
\end{aligned}
$$

Мы знаем, что для достаточно малого значения $\varepsilon>0$ и подходящего $r$

$$
\begin{aligned}
& K_{0}(1+2 \varepsilon)^{-\kappa} \mathbf{E}\left[\|\mathbf{Z}(\zeta)\|_{2}^{\kappa} l\left(\frac{\mathbf{Z}(\zeta)}{\|\mathbf{Z}(\zeta)\|_{2}}\right) I\{\zeta<\infty\}\right]-2 \varepsilon \mathbf{E}\left[\|\mathbf{Z}(\zeta)\|_{2}^{\kappa} I\{\zeta<\infty\}\right] \\
& \geqslant\left(K_{0} K(l)(1+2 \varepsilon)^{-\kappa}-2 \varepsilon\right) \mathbf{E}\left[\|\mathbf{Z}(\zeta)\|_{2}^{\kappa} I\{\zeta<\infty\}\right]>0
\end{aligned}
$$

что влечет

$$
\liminf _{y \rightarrow \infty} y^{\kappa} \mathbf{P}(\Phi>y)>0 .
$$

Последнее, в свою очередь, приводит к оценке

$$
\liminf _{y \rightarrow \infty} y^{\kappa} \mathbf{P}(\Phi>y) \geqslant K_{0} \limsup _{r \rightarrow \infty} \mathbf{E}\left[\|\mathbf{Z}(\zeta)\|_{2}^{\kappa} l\left(\frac{\mathbf{Z}(\zeta)}{\|\mathbf{Z}(\zeta)\|_{2}}\right) I\{\zeta<\infty\}\right] .
$$

Это соотношение в сочетании с (48) дает

$$
\lim _{y \rightarrow \infty} y^{\kappa} \mathbf{P}(\Phi>y)=K_{0} \lim _{r \rightarrow \infty} \mathbf{E}\left[\|\mathbf{Z}(\zeta)\|_{2}^{\kappa} l\left(\frac{\mathbf{Z}(\zeta)}{\|\mathbf{Z}(\zeta)\|_{2}}\right) I\{\zeta<\infty\}\right] \in(0, \infty) .
$$

Теорема доказана.

Теорема 5. Пусть условия теоремь 2 выполнень для докритического $(m, \varphi)$-прочесса, стартующего в момент 0 со случайного набора частии $\mathbf{Z}(0)=\left(Z_{1}(0), \ldots, Z_{m}(0)\right)$ такого, что $\mathbf{P}(\mathbf{Z}(0) \neq \mathbf{0})>0$, и случайного начального объема $\Phi(0)$ финального продукта. Если

$$
\mathbf{E}\left[\|\mathbf{Z}(0)\|^{t \vee 1}+\Phi^{t}(0)\right]<\infty
$$

для любого $t \in(0, \kappa)$, mо

$$
\mathbf{E} \Phi^{x}<\infty
$$

тогда и только тогда, когда $x \in(0, \kappa)$.

Д о к а з а т е л ь с т в о. Обозначим $\Phi_{i k}$ общий объем финального продукта, произведенного всем потомством $k$-й частицы типа $i$ нулевого поколения. Ясно, что накопленный объем $\Phi$ финального продукта можно представить в виде

$$
\Phi=\Phi(0)+\sum_{i=1}^{m} \sum_{k=1}^{Z_{i}(0)} \Phi_{i k}
$$


Для фиксированного набора $\mathbf{Z}(0)=\mathbf{z}=\left(z_{1}, \ldots, z_{m}\right)$ и $x \leqslant 1$ имеем

$$
\begin{aligned}
\mathbf{E}\left[\Phi^{x} \mid \mathbf{Z}(0)=\mathbf{z}\right] & \leqslant \mathbf{E} \Phi^{x}(0)+\sum_{i=1}^{m} \sum_{k=1}^{z_{i}} \mathbf{E} \Phi_{i k}^{x} \\
& =\mathbf{E} \Phi^{x}(0)+\sum_{i=1}^{m} z_{i} \mathbf{E}\left[\Phi^{x} \mid \mathbf{Z}(0)=\mathbf{e}_{i}\right] \\
& \leqslant \mathbf{E} \Phi^{x}(0)+\|\mathbf{z}\| \max _{1 \leqslant i \leqslant m} \mathbf{E}\left[\Phi^{x} \mid \mathbf{Z}(0)=\mathbf{e}_{i}\right] .
\end{aligned}
$$

Если же $x>1$, то существует константа $R_{x}^{*}$ такая, что (см., например, $[27$, теорема 5.2 , с. 22])

$$
\begin{aligned}
\mathbf{E}\left[\Phi^{x} \mid \mathbf{Z}(0)=\mathbf{z}\right] & \leqslant(m+1)^{x}\left[\mathbf{E} \Phi^{x}(0)+\sum_{i=1}^{m} \mathbf{E}\left(\sum_{k=1}^{z_{i}} \Phi_{i k}\right)^{x}\right] \\
& \leqslant(m+1)^{x}\left[\mathbf{E} \Phi^{x}(0)+R_{x}^{*} \sum_{i=1}^{m} z_{i}^{x} \mathbf{E}\left[\Phi^{x} \mid \mathbf{Z}(0)=\mathbf{e}_{i}\right]\right] \\
& \leqslant(m+1)^{x}\left[\mathbf{E} \Phi^{x}(0)+R_{x}^{*}\|\mathbf{z}\|^{x} \max _{1 \leqslant i \leqslant m} \mathbf{E}\left[\Phi^{x} \mid \mathbf{Z}(0)=\mathbf{e}_{i}\right]\right] .
\end{aligned}
$$

Используя формулу полной вероятности и установленные ранее оценки, получаем для $x \in(0, \kappa)$ :

$$
\begin{aligned}
\mathbf{E} \Phi^{x} & =\mathbf{P}(\mathbf{Z}(0)=\mathbf{0}) \mathbf{E} \Phi^{x}(0)+\sum_{\mathbf{z} \in \mathbb{N}_{0}^{m} \backslash\{\mathbf{0}\}} \mathbf{P}(\mathbf{Z}(0)=\mathbf{z}) \mathbf{E}\left[\Phi^{x} \mid \mathbf{Z}(0)=\mathbf{z}\right] \\
& \leqslant K_{2} \mathbf{E} \Phi^{x}(0)+K_{3} \max _{1 \leqslant i \leqslant m} \mathbf{E}\left[\Phi^{x} \mid \mathbf{Z}(0)=\mathbf{e}_{i}\right] \mathbf{E}\left[\|\mathbf{Z}(0)\|^{x \vee 1}\right]<\infty .
\end{aligned}
$$

$\mathrm{C}$ другой стороны, для любого $\mathbf{z} \in \mathbb{N}_{0}^{m} \backslash\{\mathbf{0}\}$ такого, что $\mathbf{P}(\mathbf{Z}(0)=\mathbf{z})>0$, имеем

$$
\mathbf{E} \Phi^{x} \geqslant \mathbf{E}\left[\Phi^{x} \mid \mathbf{Z}(0)=\mathbf{z}\right] \mathbf{P}(\mathbf{Z}(0)=\mathbf{z}),
$$

и необходимый результат для $x \geqslant \kappa$ следует из теоремы 2 .

8. Системы поллинга с мгновенными переключениями и $(m, \varphi)$-процессы. Мы начнем этот раздел с описания связи между эволюционирующими в случайной среде системами поллинга ветвящегося типа с финальным продуктом (сокращенно - $(P m, \varphi)$-системами) с мгновенными переключениями и $(m, \varphi)$-процессами.

Рассмотрим систему поллинга с мгновенными переключениями и предположим, что работа системы началась, когда в систему, до этого не имевшую требований, поступило требование на станцию $J \in\{1, \ldots, m\}$. Мы хотим изучить распределение периода занятости прибора, который осуществляет $J-1$ мгновенных переключений, а затем начинает обслуживание требования, прибывшего на станцию $J$.

Длина периода занятости образована интервалами времени, использованными прибором при полном обходе станций случайное количество 
раз по циклам $(1 \rightarrow 2 \rightarrow \cdots \rightarrow m \rightarrow 1)$, плюс время, необходимое для завершения последнего (может быть, неполного) цикла обслуживания, вплоть до момента, когда в системе впервые не будет требований.

Предположим, что в ходе $n$-го цикла обслуживания, входящего в первоначальный период занятости, дисциплина обслуживания на станции $i$ удовлетворяет свойству ветвления с векторнозначной с.в.п.ф.

$$
\chi_{n}(\mathbf{s} ; \lambda)=\left(\chi_{n}^{(1)}(\mathbf{s} ; \lambda), \ldots, \chi_{n}^{(m)}(\mathbf{s} ; \lambda)\right),
$$

где

$$
\chi_{n}^{(i)}(\mathbf{s} ; \lambda):=\mathbf{E}\left[s_{1}^{\theta_{i 1}(n)} \cdots s_{m}^{\theta_{i m}(n)} e^{-\lambda \phi_{i}(n)}\right], \quad i=1,2, \ldots, m .
$$

Допустим, что последовательность $\boldsymbol{\chi}_{0}(\mathbf{s} ; \lambda), \boldsymbol{\chi}_{1}(\mathbf{s} ; \lambda), \ldots$ состоит из независимых одинаково распределенных элементов. Предположим, что для каждого требования, скажем, $j$, обслуженного на станции $i$ во время $n$-го цикла, финальный продукт $\phi_{i}(n, j)$ и вектор $\left(\theta_{i 1}(n, j), \ldots, \theta_{i m}(n, j)\right)$ количества новых требований, прибывших в систему за время обслуживания $\tau_{i}(n, j)$ рассматриваемого требования, таковы, что

$$
\left(\theta_{i 1}(n, j), \ldots, \theta_{i m}(n, j) ; \phi_{i}(n, j)\right) \stackrel{d}{=}\left(\theta_{i 1}(n), \ldots, \theta_{i m}(n) ; \phi_{i}(n)\right) .
$$

Здесь в качестве финального продукта может выступать не только величина $\tau_{i}(n, j)$, но и любая неотрицательная случайная величина, либо зависящая от набора $\left(\theta_{i 1}(n, j), \ldots, \theta_{i m}(n, j) ; \tau_{i}(n, j)\right)$, либо вообще не зависящая от функционирования системы. Положим $h_{n}^{(i)}(\mathbf{s}):=\chi_{n}^{(i)}(\mathbf{s} ; 0)$, $i=1, \ldots, m$, и для $n=0,1,2, \ldots$ определим с.в.п.ф.

$$
F_{n}^{(i)}(\mathbf{s} ; \lambda)=\mathbf{E}\left[\mathbf{s}_{1}^{\xi_{i 1}(n)} \cdots \mathbf{s}_{m}^{\xi_{i m}(n)} e^{-\lambda \varphi_{i}(n)}\right]
$$

и в.п.ф.

$$
f_{n}^{(i)}(\mathbf{s})=\mathbf{E}\left[s_{1}^{\xi_{i 1}(n)} \cdots s_{m}^{\xi_{i m}(n)}\right]
$$

при помощи равенств $F_{n}^{(m)}(\mathbf{s} ; \lambda)=\chi_{n}^{(m)}(\mathbf{s} ; \lambda)$,

$$
\begin{aligned}
& \quad F_{n}^{(i)}(\mathbf{s} ; \lambda)=\chi_{n}^{(i)}\left(s_{1}, \ldots, s_{i}, F_{n}^{(i+1)}(\mathbf{s} ; \lambda), \ldots, F_{n}^{(m)}(\mathbf{s} ; \lambda) ; \lambda\right), \quad i<m, \\
& \text { и } f_{n}^{(m)}(\mathbf{s})=h_{n}^{(m)}(\mathbf{s}), \\
& \quad f_{n}^{(i)}(\mathbf{s})=h_{n}^{(i)}\left(s_{1}, \ldots, s_{i}, f_{n}^{(i+1)}(\mathbf{s}), \ldots, f_{n}^{(m)}(\mathbf{s})\right), \quad i<m .
\end{aligned}
$$

Мы хотим описать условия на системы поллинга ветвящегося типа, при выполнении которых степенные моменты объема финального продукта, накопленного в системе за период занятости, будут конечными или бесконечными. Наши результаты базируются на следующем несколько длинном, но важном утверждении, раскрывающем связь между поведением некоторых характеристик периодов занятости $(P m, \varphi)$-систем и соответствующих характеристик $(m, \varphi)$-процесса, размер популяции и объем финального продукта которого в момент 0 случайны. 
Теорема 6. Совместное распределение количества требований на различньх станииях в конце $n$-го иикла обслуживания и объем $\phi и-$ нального продукта, накопленного в системе к кониу $n$-го иикла обслуживания в $(P m, \varphi)$-системе, начинающей работу при наличии одного требования на станиии $J$ и остановленной в конче первого периода занятости, совпадает с совместным распределением числа частии в $n$-м поколении и объема финального продукта, накопленного за $n-1$ поколение в $(m, \varphi)$-прочессе, нулевое поколение которого характеризуется случайным числом частии и объемом финального продукта, подчиняюшимися с.в.п.ф. $F_{0}^{(J)}(\mathbf{s} ; \lambda)$, и в котором совместное распределение числа непосредственных потомков и объем финального продукта, произведенного частичами различных типов $k$-го поколения, задается векторнозначными с.в.n.ф.

$$
\mathbf{F}_{k}(\mathbf{s} ; \lambda)=\left(F_{k}^{(1)}(\mathbf{s} ; \lambda), \ldots, F_{k}^{(m)}(\mathbf{s} ; \lambda)\right), \quad k=1,2, \ldots, n .
$$

Для доказательства данной теоремы необходимо почти дословно повторить доказательство теоремы 4 работы [36], поэтому мы опускаем соответствующие рассуждения.

Заметим, что если первоначально вместо одного требования на станции $J$ в системе имеется набор требований $\left(k_{1}, \ldots, k_{m}\right)$, где $k_{i}-$ количество требований на станции $i$, то распределение первоначального числа частиц и начальный объем финального продукта в соответствующем $(m, \varphi)$-процессе следует задавать с.в.п.ф.

$$
F_{0}(\mathbf{s} ; \lambda)=\prod_{J=1}^{m}\left(F_{0}^{(J)}(\mathbf{s} ; \lambda)\right)^{k_{J}} .
$$

Будем называть $(m, \varphi)$-процесс, описанный в теореме 6 , ассоциированньлм $(m, \varphi)$-процессом для соответствующей $(P m, \varphi)$-системы.

Теперь мы можем переформулировать результаты разд. 3 в терминах нашей системы поллинга.

Пусть $A_{n}:=\left(a_{i j}(n)\right)_{i, j=1}^{m}$ - матрица с элементами

$$
a_{i j}(n):=\left.\frac{\partial}{\partial s_{j}} f_{n}^{(i)}(\mathbf{s})\right|_{\mathbf{s}=\mathbf{1}}=\mathbf{E}_{\mathbf{f}} \xi_{i j}(n),
$$

a $H_{n}:=\left(h_{i j}(n)\right)_{i, j=1}^{m}$ - матрица с элементами

$$
h_{i j}(n):=\left.\frac{\partial}{\partial s_{j}} h_{n}^{(i)}(\mathbf{s})\right|_{\mathbf{s}=\mathbf{1}}=\mathbf{E}_{\mathbf{h}} \theta_{i j}(n) .
$$

В силу $(51) a_{m j}(n)=h_{m j}(n), j=1, \ldots, m$, и при $i<m$

$$
a_{i j}(n)=h_{i j}(n) I\{j \leqslant i\}+\sum_{k=i+1}^{m} h_{i k}(n) a_{k j}(n) .
$$


Для $i=1, \ldots, m$ введем вспомогательные матрицы

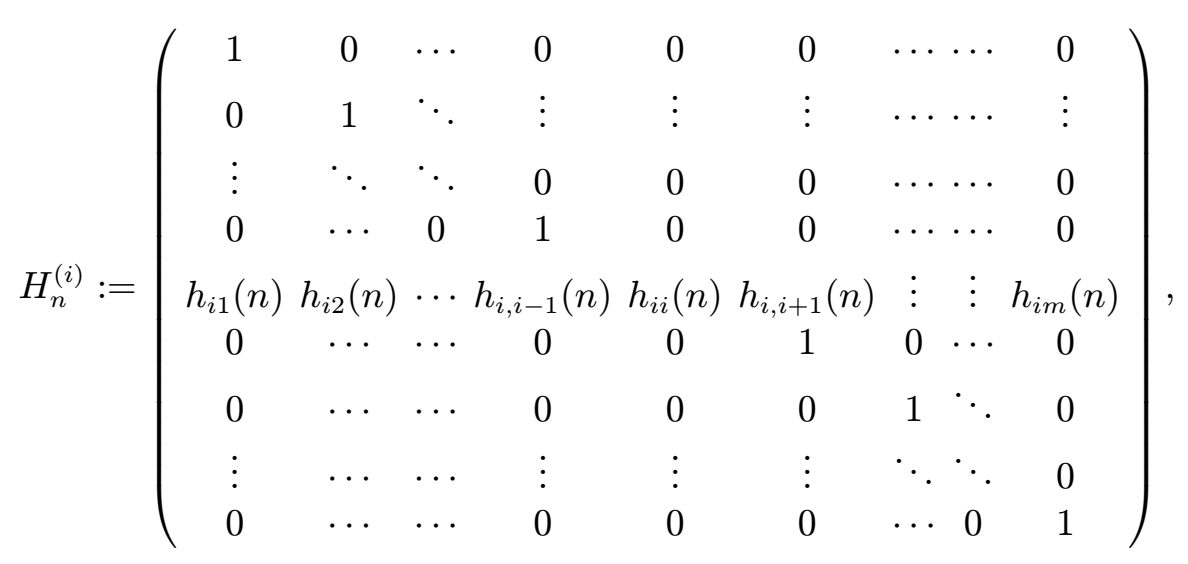

где при любом $i$ элементы матрицы $H_{n}$ расположены только в строке $i$ матрицы $H_{n}^{(i)}$. При помощи (53) нетрудно проверить, что

$$
A_{n}=H_{n}^{(1)} \cdots H_{n}^{(m)} .
$$

Далее, пусть $\mathbf{C}_{n}:=\left(C_{1}(n), \ldots, C_{m}(n)\right)^{\prime}-$ случайный вектор с компонентами

$$
C_{i}(n):=\left.\frac{d}{d \lambda} F_{n}^{(i)}(\mathbf{1} ; \lambda)\right|_{\lambda=0}=\mathbf{E}_{\mathbf{F}} \varphi_{i}(n), \quad i=1, \ldots, m,
$$

и пусть $\mathbf{c}_{n}:=\left(c_{1}(n), \ldots, c_{m}(n)\right)^{\prime}-$ случайный вектор с компонентами

$$
c_{i}(n):=\left.\frac{d}{d \lambda} \chi_{n}^{(i)}(\mathbf{1} ; \lambda)\right|_{\lambda=0}=\mathbf{E}_{\boldsymbol{\chi}} \phi_{i}(n), \quad i=1, \ldots, m .
$$

В силу $(50) C_{m}(n)=c_{m}(n)$ и для $i<m$

$$
C_{i}(n)=c_{i}(n)+\sum_{k=i+1}^{m} h_{i k}(n) C_{k}(n) .
$$

Отсюда вытекает, что $\mathbf{C}_{n}=\mathbf{c}_{n}+H_{n}^{\Delta} \mathbf{C}_{n}$, где

$$
H_{n}^{\Delta}:=\left(h_{i j}(n) I\{i<j\}\right)_{i, j=1}^{m}
$$

- верхне-треугольная матрица, порожденная матрицей $H_{n}$. Таким образом, $\mathbf{C}_{n}=\left(E-H_{n}^{\Delta}\right)^{-1} \mathbf{c}_{n}$.

Следующие два утверждения являются простыми следствиями теорем 5 и 6.

В первой теореме указаны условия, при которых период занятости $(P m, \varphi)$-системы бесконечен с положительной вероятностью. 
Теорема 7. Предположим, что $(m, \varphi)$-прочесс, ассочиированньии c $(P \mathrm{P}, \varphi)$-системой, таков, что подчиненньй ему ВПСС удовлетворяет условиям теоремы 1 с $\alpha>0$ и, кроме того, выполнено условие (16). Если $\Phi$ - общий объем финального продукта, накопленного в $($ Pm, $\varphi)$-системе за период занятости, то $\mathbf{P}(\Phi=\infty)>0$. В частности, если время обслуживания любого требования положительно с вероятностью 1, то период занятости $(P m, \varphi)$-системь бесконечен $c$ положительной вероятностью.

Формулировка результатов для $(P m, \varphi)$-системы с докритическим ассоциированным $(m, \varphi)$-процессом требует больше усилий.

Теорема 8. Предположим, что $(m, \varphi)$-прочесс, ассоииированньии c $($ Рт, $)$-системой, является докритическим, удовлетворяет условиям теоремь 2 и

$$
\min _{1 \leqslant J \leqslant m} F_{0}^{(J)}(\mathbf{0} ; 0)>0 \text {. }
$$

Если параметр к, задаваемьий равенством (20), таков, что

$$
\max _{1 \leqslant J \leqslant m} \mathbf{E}\left[\left(\xi_{J 1}+\cdots+\xi_{J m}\right)^{t \vee 1}+\varphi_{J}^{t}(n)\right]<\infty
$$

для любого $t \in(0, \kappa)$, то найдется константа $C \in(0, \infty)$ такая, что

$$
\mathbf{P}(\Phi>y) \sim C y^{-\kappa}, \quad y \rightarrow \infty .
$$

В частности, если финальньл продуктом требования является время его обслуживания, то хвост распределения длинь $\Phi$ периода занятости системь удовлетворяет асимптотическому соотношению (55).

Следствие 1. Если вьполнень условия теоремь 8, то $\mathbf{E} \Phi^{x}<\infty$ тогда и только тогда, когда $x \in(0, \kappa)$.

Вернемся теперь к примерам 1 и 2, рассматривавшимся в начале статьи.

Дифференцируя соотношение (2) в точке $\mathbf{s}=\mathbf{1}$, получаем, что матрица $H_{n}$ и вектор $\mathbf{C}_{n}$ в примере 1 имеют элементы

$$
h_{i j}(n)=\gamma_{i j}(n)+\varepsilon_{i j}(n) \mathbf{E}\left[\tau_{i}(n) \mid T_{i n}\right], \quad i, j=1, \ldots, m,
$$

И

$$
C_{i}(n)=\mathbf{E}\left[\tau_{i}(n) \mid T_{i n}\right], \quad i=1, \ldots, m,
$$

в то время как дифференцирование равенства (5) в точке $\mathbf{s}=\mathbf{1}$ с учетом соотношения (3) и очевидных преобразований показывает, что $h_{i i}(n)=0$, $i=1, \ldots, m$, а при $i \neq j$

$$
h_{i j}(n)=\frac{\gamma_{i j}(n)\left(1-\gamma_{i i}(n)\right)+\varepsilon_{i j}(n) \mathbf{E}\left[\tau_{i}(n) \mid T_{i n}\right]}{1-\gamma_{i i}(n)-\varepsilon_{i i}(n) \mathbf{E}\left[\tau_{i}(n) \mid T_{i n}\right]}
$$


если

$$
\frac{1-\gamma_{i i}(n)}{\mathbf{E}\left[\tau_{i}(n) \mid T_{i n}\right]}>\varepsilon_{i i}(n)
$$

и $h_{i j}(n)=\infty$ в противном случае.

Заметим, что если величины $\varepsilon_{i j}(n), i, j=1, \ldots, m$, неслучайны, $\gamma_{i i}(n)=0$ с вероятностью 1 для всех $i=1, \ldots, m$, а $T_{i n}$ - экспоненциальное распределение со случайным параметром $\mu_{i n}$, то соотношения (56) и (57) выглядят следующим образом:

$$
h_{i j}(n)=\frac{\mu_{i n} \gamma_{i j}(n)+\varepsilon_{i j}(n)}{\mu_{i n}-\varepsilon_{i i}(n)}
$$

и

$$
\mu_{i n}-\varepsilon_{i i}(n)>0 .
$$

Эти значения находятся в полном согласии с соответствующими формулами и ограничениями, приведенными в разделах 1.1 и 1.2 работы [34].

Заключительные замечания. Наши результаты дают критерий, позволяющий ответить на вопрос: когда величина $\Phi-$ общий объем финального продукта, накопленного в системе поллинга с мгновенными переключениями за период занятости, имеет конечный или бесконечный момент порядка $x$ ? С этой точки зрения теорема 8 уточняет и обобщает в нескольких направлениях теорему 1.1 статьи [34]. Например, мы не требуем экспоненциальности распределений времен обслуживания требований и устанавливаем упомянутый критерий для широкого класса систем поллинга, не затрагиваемых результатами работы [34]. Более того, мы даже описываем поведение хвоста распределения случайной величины $\Phi$. K сожалению, такого рода уточнение достигается за счет усложнения накладываемых условий. Наиболее существенным из наших предположений является условие Т, выполнение которого установлено к настоящему моменту только для ограниченного класса неотрицательных случайных матриц. Расширение класса неотрицательных случайных матриц, подчиняющихся условию Т, является интересной и трудной задачей. Некоторые утверждения, связанные с этим кругом проблем, были получены совсем недавно в целом ряде работ (см., например, [17] и [26]). Однако эти результаты не применимы к случаю мер, полностью сконцентрированных на неотрицательных матрицах.

\section{СПИСОК ЛИТЕРАТУРЫ}

1. Альбеверио C. А., Козлов М.В. О возвратности и транзиентности зависящих от состояния ветвящихся процессов в случайной среде. - Теория вероятн. и ее. примен., 2003, т. 48, в. 4, с. 641-660. 
2. Ватутин B. А., Дьяконова E. E. Ветвящиеся процессы Гальтона-Ватсона в случайной среде. I: Предельные теоремы. - Теория вероятн. и ее. примен., 2003, т. 48 , в. 2 , с. $274-300$.

3. Ватутин В. А., Дьяконова Е. Е. Ветвящиеся процессы Гальтона-Ватсона в случайной среде. II: Конечномерные распределения. - Теория вероятн. и ее. примен., 2004, т. 49, в. 2, с. 231-268.

4. Вишневский В. М., Семенова О. В. Математические методы исследования систем поллинга. - Автоматика и телемеханика, 2006, т. 2, с. 3-56.

5. Гришечкин C. А. Одноканальные системы с круговым доступом или разделением процессора и ветвящиеся процессы. - Матем. заметки, 1988, т. 44, с. 433-448.

6. Гришечкин C. А. Ветвящиеся процессы и системы с повторными вызовами или случайной дисциплиной. - Теория вероятн. и ее. примен., 1990, т. 35, в. 1, с. 3550.

7. Козлов М. В. Условная предельная теорема критического ветвящегося процесса в случайной среде. - Докл. РАН, 1995, т. 344, № 1, с. 12-15.

8. Севастьянов Б. А. Ветвящиеся процессы. М.: Наука, 1971, 436 с.

9. Яшков С. Ф., Яшкова А.С. Разделение процессора: обзор математической теории. - Информационные системы, 2007, т. 7, № 3, с. 248-322.

10. Afanasyev V.I., Geiger J., Kersting G., Vatutin V.A. Criticality for branching processes in random environment. - Ann. Probab., 2005, v. 33, № 2, p. 645-673.

11. Afanasyev V.I., Geiger J., Kersting G., Vatutin V.A. Functional limit theorems for strongly subcritical branching processes in random environment. - Stoch. Process. Appl., 2005, v. 115, № 10, p. 1658-1676.

12. Altman E. Semi-linear stochastic difference equations. — Discrete Event Dyn. Syst., 2009, v. 19, p. 115-136.

13. Altman E., Fiems D. Expected waiting time in symmetric polling systems with correlaite walking times. - Queueing Syst., 2007, v. 56, p. 241-253.

14. Athreya K.B., Karlin S. On branching processes with random environments. I: Extinction probability. - Ann. Math. Statist., 1971, v. 42, p. 1499-1520.

15. Athreya K. B., Karlin S. On branching processes with random environments. II: Limit theorems. - Ann. Math. Statist., 1971, v. 42, p. 1843-1858.

16. Athreya K. B., Ney P. E. Branching Processes. Berlin: Springer-Verlag, 1972, 288 p.

17. Buraczewski D., Damek E., Guivarc'h Y., Hulanicki A., Urban R. Tail-homogeneity of stationary measures for some multidimensional stochastic recursions. - Probab. Theory Related Fields, 2009, v. 145, № 3-4, p. 385-420.

18. Davis R.A., Mikosch T., Basrak B. Limit theory for the sample autocorrelations of solutions to stochastic recurrence equations with applications to GARCH processes. Preprint, 1999.

19. Dembo A., Peres Y., Zeitouni O. Tail estimates for one-dimensional random walk in random environment. - Comm. Math. Phys., 1996, v. 181, p. 667-683.

20. Dyakonova E., Geiger J., Vatutin V. On the survival probability and a functional limit theorem for branching processes in random environment. - Markov Process. Related Fields, 2004, v. 10, p. 289-306.

21. Foss $S$., Chernova $N$. On the stability of a partially accessible multi-station queue with state-dependent routing. - Queueing Systems Theory Appl., 1998, v. 29, № 1, p. $55-73$.

22. Foss S., Kovalevskii A. A stability criterion via fluid limits and its application to a polling system. - Queueing Systems Theory Appl., 1999, v. 32, № 1, p. 131-168.

23. Foss S., Last G. On the stability of greedy polling systems with general service policies. - Probab. Eng. Inform. Sci., 1998, v. 12, № 1, p. 49-68.

24. Fuhrmann S.W. A decomposition result for a class of polling models. - Queueing Systems Theory Appl., 1992, v. 11, № 1-2, p. 109-120.

25. Geiger J., Kersting G. The survival probability of a critical branching process in random environment. - Теория вероятн. и ее примен., 2000, т. 45, в. 3, с. 607-615.

26. Guivarc'h $Y$. Heavy tail properties of stationary solutions of multidimensional stochastic recursions. - Dynamics \& Stochastics. Beachwood: Inst. Math. Statist., 2006, p. 85-99. (IMS Lecture Notes Monogr. Ser., v. 48.)

27. Gut A. Stopped Random Walks. Limit Theorems and Applications. New York: 
Springer-Verlag, 1988, $198 \mathrm{p}$.

28. Kesten $H$. Random difference equations and renewal theory for products of random matrices. - Acta Math., 1973, v. 131, p. 207-248.

29. Kesten H., Kozlov M. V., Spitzer F. A limit law for random walk in a random environment. - Compositio Math., 1975, v. 30, № 2, p. 145-168.

30. Key E. Computable examples of the maximal Lyapunov exponent. - Probab. Theory Related Fields, 1987, v. 75, № 1, p. 97-107.

31. Kingman J. F. C. Subadditive ergodic theory. - Ann. Probab., 1973, v. 1, p. 883-909.

32. MacPhee I., Menshikov M., Popov S., Volkov S. Periodicity in the transient regime of exaustive polling systems. - Ann. Appl. Probab., 2006, v. 16, № 4, p. 1816-1850.

33. MacPhee I., Menshikov M., Petritis D., Popov S. A Markov chain model of a polling systems with parameter regeneration. - Ann. Appl. Probab., 2007, v. 17, № 5-6, p. $1447-1473$.

34. MacPhee I., Menshikov M., Petritis D., Popov S. Polling systems with parameter regeneration, the general case. - Ann. Appl. Probab., 2008, v. 18, №6, p. 21312155 .

35. van der Mei R.D. Towards a unifying theory of branching-type polling systems in heavy traffic. - Queueing Systems Theory Appl., 2007, v. 57, № 1, p. 29-46.

36. Resing J. A. C. Polling systems and multitype branching processes. - Queueing Systems Theory Appl., 1993, v. 13, № 4, p. 409-426.

37. Smith W. L., Wilkinson W.E. On branching processes in random environments. Ann. Math. Statist., 1969, v. 40, p. 814-827.

38. Tanny D. On multitype branching processes in a random environment. - Adv. Appl. Probab., 1981, v. 13, № 3, p. 464-497.

39. Vatutin V. A., Zubkov A. M. Branching Processes. II. - J. Soviet Math., 1993, v. 67, p. 3407-3485.

40. Vatutin V.A., Dyakonova E.E. Reduced branching processes in random environment. - Mathematics and Computer Science. II: Algorithms, Trees, Combinatorics and Probabilities. Ed. by B. Chauvin et al. Basel: Birkhäuser, 2002, p. 455-467.

41. Vatutin V.A., Dyakonova E. E. Yaglom type limit theorem for branching processes in random environment. - Mathematics and Computer Science. III: Algorithms, Trees, Combinatorics and Probabilities. Ed. by M. Drmota et al. Basel: Birkhäuser, 2004, p. $375-385$.

42. Vatutin V.A., Dyakonova E.E. Multitype branching processes and some queueing systems. - J. Math. Sci., 2002, v. 111, № 6, p. 3901-3909.

Поступила в редакцию

21.X.2009

Исправленный вариант 26.IV.2010 